\title{
The Effect of Acoustic Forcing on Instabilities and Breakdown in Swept-Wing Flow Over a Backward-Facing Step
}

\author{
Jenna L. Eppink* \\ NASA Langley Research Center, Hampton, VA 23681 \\ Olga Shishkov ${ }^{\dagger}$ \\ Georgia Institute of Technology, Atlanta, GA, 30332 \\ Richard W. Wlezien ${ }^{\ddagger}$ \\ Iowa State University, Ames, IA, 50011 \\ Rudolph A. King*, Meelan Choudhari ${ }^{\S}$ \\ NASA Langley Research Center, Hampton, VA 23681
}

\begin{abstract}
Instability interaction and breakdown were experimentally investigated in the flow over a swept backward-facing step. Acoustic forcing was used to excite the Tollmien-Schlichting (TS) instability and to acquire phase-locked results. The phase-averaged results illustrate the complex nature of the interaction between the TS and stationary crossflow instabilities. The weak stationary crossflow disturbance causes a distortion of the TS wavefront. The breakdown process is characterized by large positive and negative spikes in velocity. The positive spikes occur near the same time and location as the positive part of the TS wave. Higher-order spectral analysis was used to further investigate the nonlinear interactions between the TS instability and the traveling crossflow disturbances. The results reveal that a likely cause for the generation of the spikes corresponds to nonlinear interactions between the TS, traveling crossflow, and stationary crossflow disturbances. The spikes begin at low amplitudes of the unsteady and steady disturbances $\left(2-4 \% U_{e}\right)$ but can achieve very large amplitudes $\left(20-30 \% U_{e}\right)$ that initiate an early, though highly intermittent, breakdown to turbulence.
\end{abstract}

\section{Nomenclature}

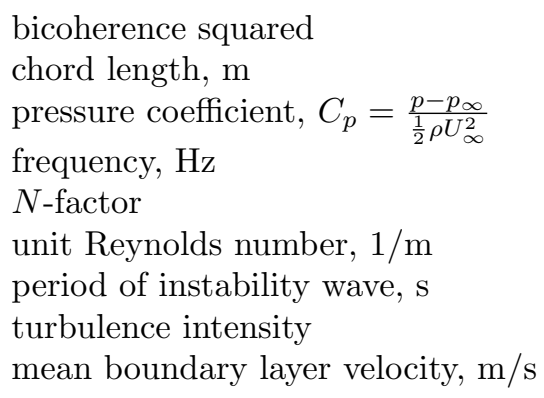

${ }^{*}$ Research Aerospace Engineer, Flow Physics and Control Branch, M.S. 170, AIAA Member

${ }^{\dagger}$ Graduate Student, George W. Woodruff School of Mechanical Engineering, 801 Ferst Drive

${ }_{\ddagger}$ Professor and Vance and Arlene Coffman Endowed Department Chair in Aerospace Engineering, 2271 Howe Hall, Room 1200A, AIAA Fellow.

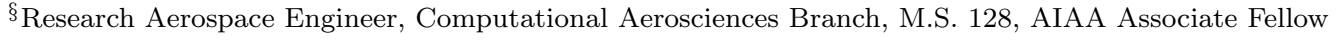




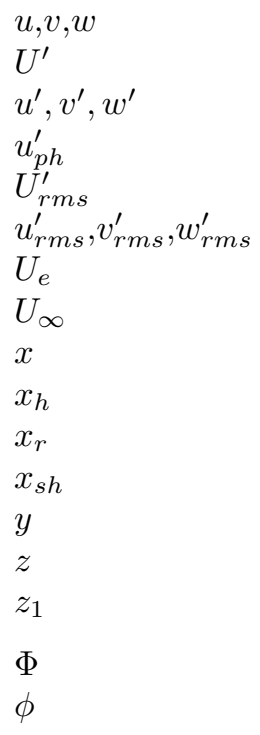

BFS

DRE

LFC

TCF

TS velocity components in the $x, y$, and $z_{n}$ directions, $\mathrm{m} / \mathrm{s}$

steady disturbance velocity, $\mathrm{m} / \mathrm{s}$

fluctuating components of velocity, $\mathrm{m} / \mathrm{s}$

instantaneous phase averaged velocity, $\mathrm{m} / \mathrm{s}$

spanwise root mean square of steady disturbance velocity, $U^{\prime}, \mathrm{m} / \mathrm{s}$

temporal root mean square of $u^{\prime}, v^{\prime}, w^{\prime}, \mathrm{m} / \mathrm{s}$

boundary layer edge velocity, $\mathrm{m} / \mathrm{s}$

freestream velocity, $\mathrm{m} / \mathrm{s}$

streamwise direction

streamwise location of step

reattachment location in terms of the number of step heights downstream of the step

number of step heights downstream of step in the $x$-direction

wall-normal direction

spanwise direction (parallel to the leading edge)

initial spanwise location of $x y$-scan, mm

biphase, rad

phase, rad

Backward-facing Step

Discrete Roughness Element

Laminar Flow Control

Traveling-Crossflow

Tollmien-Schlichting

\section{Introduction}

Improving aircraft fuel efficiency is the ultimate goal of many aerospace science research efforts. Cuts in airplane fuel consumption will not only help keep airline flights at reasonable costs despite the ever-increasing price of fuel but can also result in significant reductions in $\mathrm{CO}_{2}$ emissions. One promising approach, which can contribute significantly to the overall vehicle drag reduction, is to utilize laminar flow control (LFC) techniques, either passive or active, to maintain laminar flow over large portions of the aircraft surface (the wings in particular).

In theory and in the laboratory, LFC techniques can work quite well. However, real-world applications result in additional difficulties that can be detrimental if not well-managed. One such difficulty is the disruption of laminar flow that can occur if small protuberances or surface imperfections are present on the wing surface. These imperfections can result from insect residue, rivets, bolts, steps, gaps, paint, and other sources. In order for LFC to be effective in an operational environment, it is crucial that we gain a better understanding of how surface irregularities affect transition. This is important so that more reasonable manufacturing tolerances can be specified.

In a swept-wing flow, stationary crossflow is typically the dominant transition mechanism. However, when a step is introduced into the flow, the step can act as a receptivity site for other types of disturbances, and it can also modify the mean flow so that those disturbances are destabilized for a short region downstream of the step.

The effect of 2D steps on swept-wing transition has been studied recently. This work has generally been limited to observing the behavior of the transition front as the step height is increased, ${ }^{1,2}$ but more recently, researchers have begun to study the flow in more detail. Duncan et al. ${ }^{3}$ performed hotwire measurements downstream of forward- and backward-facing steps to determine the effect of the steps on stationary crossflow instabilities. They found that the steps caused an increase in N-factor for the stationary crossflow, but the stationary crossflow amplitudes were very low at the step, and therefore the uncertainty of the N-factors was high. Tufts et al. ${ }^{4}$ performed computations to study the interaction between stationary crossflow instabilities and a two-dimensional step excrescence. They found that the backward-facing step did not amplify the stationary crossflow modes, but they did verify the existence of a traveling instability in the recirculation region downstream of the step. They conclude that transition is likely caused by an interaction between this traveling mode and the stationary crossflow mode. Eppink et al. ${ }^{5}$ performed detailed boundary-layer measurements downstream of a backward-facing step and identified several different types of instabilities 
that were introduced by the step and modulated by the stationary crossflow vortices. One of the unsteady disturbances identified downstream of the step is believed to be a traveling crossflow instability, similar to what was seen in the computations of Tufts et al. ${ }^{4}$ The second type of instability identified downstream of the step was a nearly two-dimensional, i.e., streamwise traveling disturbance, which, based on the mode shape, phase speed, and wave angle, is believed to be a Tollmien-Schlichting (TS) instability.

The interaction of stationary crossflow and TS disturbances is not well understood and, in general, has not been studied in much detail. Bassom and $\mathrm{Hall}^{6}$ performed a theoretical study of the interaction of stationary crossflow and TS disturbances on a rotating disc. They found that the main influence of the stationary crossflow vortex on the TS wave was through the mean-flow modification caused by the stationary crossflow vortex. The vortex could have either a stabilizing or a destabilizing effect depending on the orientation of the TS wave. The measurements by Fransson et al. ${ }^{7}$ and simulations of Choudhari and Fischer ${ }^{8}$ illustrate the differential spanwise growth of TS modes in the presence of stationary streaks (analagous to crossflow vortices) in 2-D boundary layers. Wassermann and Kloker ${ }^{9}$ performed computations to study laminar breakdown in the adverse pressure gradient of a swept flat-plate flow, with varying TS and stationary crossflow relative amplitudes. The stationary crossflow modes in this case result from the upstream region of favorable pressure gradient flow on the flat plate. They found that the interaction of the stationary crossflow and TS instabilities results in the generation of fundamental low-frequency secondary modes, but the TS waves themselves are not important for the growth of these modes nor for breakdown.

Transition over protuberances (such as 2D steps) is complicated and apparently involves the interaction of multiple types of disturbances. The current research is a follow-on experiment to the experiment described by Eppink et al. ${ }^{5}$ Acoustic forcing is used in an attempt to answer some remaining questions pertaining to the types of instabilities that occur downstream of the step, as well as how the different instabilities interact and ultimately lead to breakdown. Sections II-III cover the background of the experimental setup and previously reported results. The general effect of forcing on the different instability mechanisms is discussed in Section IV. The phase-averaged results showing the interaction of the TS and stationary crossflow instabilities are then presented in Section V, and the "spike" breakdown mechanism is discussed in Section VI. Finally, results from the higher-order spectral analysis are discussed in Section VII.

\section{Experimental Setup}

The experiment was performed in the 2 Foot by 3 Foot Low-Speed Boundary-Layer Channel at NASA Langley Research Center. The tunnel is a closed circuit facility with a $0.61 \mathrm{~m}$ high by $0.91 \mathrm{~m}$ wide by 6.1 $\mathrm{m}$ long test section. The tunnel can reach speeds up to $45 \mathrm{~m} / \mathrm{s}\left(R e^{\prime}=2.87 \times 10^{6} / \mathrm{m}\right)$ in the test section. Freestream turbulence intensity levels, $T u=\frac{1}{U_{\infty}} \sqrt{\frac{1}{3}\left(u^{\prime 2}+v^{\prime 2}+w^{\prime 2}\right)}$, were measured using a crosswire in an empty test section to be less than $0.06 \%$ for the entire speed range of the tunnel, and less than $0.05 \%$ for the test speed of $26.5 \mathrm{~m} / \mathrm{s}$. This value represents the total energy across the spectrum (from $0.25 \mathrm{~Hz}$ to 10 $\mathrm{kHz}$ ), and has not been filtered to remove the low-frequency acoustic component. Thus, this tunnel can be considered a low-disturbance facility for purposes of conducting transition experiments. ${ }^{10}$

The $0.0127-\mathrm{m}$ thick flat plate model consists of a $0.41 \mathrm{~m} \mathrm{long}, 30^{\circ}$ swept leading-edge piece and a longer downstream piece (see Fig. 1). The model is $0.91 \mathrm{~m}$ wide (thus, spanning the width of the test section) and $2.54 \mathrm{~m}$ long on the longest edge. The downstream or leading-edge pieces can be adjusted relative to each other using precision shims to create either a forward-facing or a backward-facing 2D step of a desired height that is parallel with the leading edge. The leading-edge piece was polished to a surface finish of $0.2 \mu \mathrm{m}$, and the larger downstream plate had a surface finish of $0.4 \mu \mathrm{m}$. The chord, $c$, is taken as the longest edge of the plate $(2.54 \mathrm{~m})$ and is used to non-dimensionalize quantities throughout the paper. Thus, the step is located at $x / c=0.161$. A leading-edge contour was designed for the bottom side of the plate to make the suction-peak less severe and avoid separation, which could potentially cause unsteadiness in the attachment-line flow.

A 3D pressure body along the ceiling was designed to induce a streamwise pressure gradient so that transition would be dominated by stationary crossflow. The ceiling liner was contoured to simulate nearly infinite swept-wing flow within a mid-span measurement region of width 0.3 meters. This was achieved by designing the liner such that the $C_{p}$ contours were parallel with the leading edge within the core region. The ceiling liner was fabricated out of a hard foam using a computer-controlled milling machine.

All measurements were performed at a freestream velocity of $26.5 \mathrm{~m} / \mathrm{s}\left(R e^{\prime}=1.69 \times 10^{6} / \mathrm{m}\right)$. The data were acquired using a hotwire mounted on a traversing system that could be moved in all three $(x, y$, and $z$ ) directions. Detailed boundary-layer measurements allowed for tracking of the instability growth and 
determining the effect of the backward-facing step on the instabilities. Additionally, sublimating-chemical flow visualization was performed using naphthalene to determine transition location. Discrete roughness elements (DREs) were used to excite the most-amplified stationary crossflow wavelength. The DREs were applied with a spanwise spacing, $\lambda_{z}$, of $11 \mathrm{~mm}$ and were approximately $20 \mu \mathrm{m}$ thick. DREs with two different diameters were used: $2.75 \mathrm{~mm}$ (small DREs) and $4.4 \mathrm{~mm}$ (large DREs). The large diameter DREs led to larger initial amplitudes of the stationary crossflow due to the enhanced receptivity. The spacing of the DREs $(11 \mathrm{~mm})$ corresponds to the most amplified stationary crossflow wavelength calculated for the baseline case (i.e., with no step).

Hotwire data were obtained using a constant-temperature anemometer with a 1:1 bridge configuration. Single-sensor hotwire probes were used throughout the test for the boundary-layer measurements. The wires were 5 - $\mu \mathrm{m}$ diameter, platinum-plated tungsten wires with a sensor length of $1.25 \mathrm{~mm}$. The data were ACcoupled at $0.25 \mathrm{~Hz}$ to acquire the fluctuating velocity component and low-pass filtered at a cut-off of $10 \mathrm{kHz}$. Data were typically digitized at a sampling rate of $25 \mathrm{kHz}$. For more details of the experiment setup, as well as data reduction and uncertainty analysis, refer to Eppink et al. ${ }^{5}$

The acoustic excitation was provided by four speakers that were flush mounted on the wall upstream of the test section. The speakers were mounted with the cones normal to the flow direction. The 8-Ohm, 203.3-mm diameter woofers have a frequency range of 30 to $500 \mathrm{~Hz}$.

\section{Previous Experimental Results}

Results for the backward-facing step (BFS) cases are discussed in detail by Eppink et al. ${ }^{5}$ but will be summarized here as context for the following discussion. Transition occurred far downstream and was dominated by stationary crossflow until the step height was increased above approximately $49 \%$ of the local boundary-layer thickness for the baseline case. At that step height, transition moved upstream abruptly, but still occurred more then 300 step heights downstream of the step. This was also well downstream of the separation bubble, which extended approximately 30 step heights downstream of the step. The velocity spectra downstream of the step were found to be rich with unsteady disturbances in a broad frequency band $(f \approx 80$ to $1500 \mathrm{~Hz})$. These unsteady disturbances were not present in the baseline case and are believed to be directly responsible for transition in the presence of the step since the stationary crossflow amplitudes remained too low to cause transition via their high-frequency secondary instabilities. Data were acquired simultaneously from two hotwires in the boundary layer to extract phase speed and wave angle information. Based on these results, three distinct disturbance bands were identified corresponding to a traveling crossflow (TCF) type disturbance $(f=80$ to $200 \mathrm{~Hz})$, a TS-like disturbance $(f=200$ to $800 \mathrm{~Hz})$, and a shear-layer disturbance $(f=800$ to $1500 \mathrm{~Hz})$. The linear stability calculations performed for the baseline case did not show the existence of any unstable TS modes due to the favorable pressure gradient. The existence of this instability in the experiment is believed to be due to the short unfavorable pressure gradient region that exists downstream of the BFS. The TS instabilities may be unstable in this short region, and then persist downstream due to nonlinear interactions.

The unsteady disturbances were modulated in the spanwise direction by the stationary crossflow and thus formed regions of peaks and valleys in amplitude with spanwise spacing corresponding to the dominant stationary crossflow wavelength. The initial amplitude of the stationary crossflow affected the peak amplitude of the unsteady disturbances across all three bands, causing the amplitudes to increase with increasing initial amplitude, and thus causing transition to move upstream. Thus, the effect of stationary crossflow on transition in the backward-facing step case is understood to be via the spanwise modulation and increased growth of the unsteady disturbances. Large spikes in velocity were observed to occur well upstream of the breakdown location and appeared to be related to some type of breakdown mechanism resulting from the unsteady disturbances.

\section{General Effect of Sound on Unsteady Disturbances}

All measurements with acoustic forcing were performed with a BFS height of $45 \%$ of the local boundarylayer thickness and with the large DREs. This step height was just below the critical step height of $49 \%$, where transition moved upstream abruptly from the baseline case, but the unsteady disturbances were still present at this step height and intermittent breakdown was beginning to occur. This lower step height was chosen for the current investigation because it was believed that the more intermittent nature of the spikes 
would make them easier to identify and study.

Limited measurements were performed initially to determine the effect of acoustic forcing in each of the three disturbance bands identified in the case without such forcing. It was found that forcing in the traveling crossflow frequency band did not result in any measurable change in boundary layer disturbances across any band. Forcing in the TS and shear-layer frequency bands resulted in increased disturbance amplitudes across a broad range of frequencies. These results are not surprising given the current understanding of the different disturbance types. Traveling crossflow modes are not expected to be sensitive to acoustic forcing. ${ }^{11-13}$ However, TS and shear-layer instabilities are sensitive to sound. ${ }^{14-21}$

Initially, yz-scans spanning a short spanwise segment of approximately 1 stationary crossflow wavelength were performed at three different streamwise locations with and without forcing in the TS frequency band $(f=260 \mathrm{~Hz})$. The forcing signal was measured in the freestream to be approximately $94 \mathrm{~dB}$. The steady disturbance velocity contours are shown in Fig. 2. There are some minor differences evident in these scans, but there does not appear to be any major difference between the forced and unforced cases. Certainly, the forcing does not cause any significant change in amplitude of the stationary crossflow. The $u^{\prime}$ rms amplitude in each disturbance band at $x_{s h}=100$ is shown in Fig. 3. The mean-flow contours are also overlaid on these plots. The modulation of the unsteady disturbances due to the action of the stationary crossflow is evident in this figure. It is also apparent that the acoustic forcing results in increased peak amplitudes in all three of the disturbance bands. This is illustrated further in Fig. 4, in which the difference in the peak amplitudes between the forced and unforced cases from each yz-scan are compared. The peak TS amplitude with the forcing is significantly larger than with no forcing at the two upstream measurement locations. This increase in amplitude is expected since the forcing frequency is within the TS frequency range. There is only a very small difference between the forced and unforced cases in the traveling crossflow frequency range at the most upstream measurement location. However, the two downstream locations exhibit a larger amplitude difference, indicating that the forced case results in larger peak amplitudes even in this frequency band. The shear-layer instability behaves similarly to the traveling crossflow instability. This increase in amplitude across the traveling-crossflow and shear-layer instability frequency bands is interesting given that the forcing is confined to the TS frequency. This behavior indicates that the TS disturbances are undergoing nonlinear interactions with disturbances in the other frequency bands.

The effects of forcing on velocity spectra at a single yz location at each measurement station are compared in Fig. 5. The peak at $260 \mathrm{~Hz}$ in Figs. 5a and 5b corresponds to the forcing frequency. As expected, at $x_{s h}=65$ the effect of forcing on TS disturbances is limited to a narrow frequency band near the forcing frequency. The increase in the traveling-crossflow frequency range $(80-200 \mathrm{~Hz})$ with forcing is evident at the two downstream locations, $x_{s h}=100$ and 145 . The increase in the shear-layer instability portion of the frequency spectrum $(800-1500 \mathrm{~Hz})$ first becomes noticeable at $x_{s h}=145$, even though Fig. 4 shows an increase in the peak integrated amplitude of this frequency band at $x_{s h}=100$. This discrepancy is likely due to the fact that these spectra were taken at a single location near the peak of the TS instability, and the peak location of the shear-layer disturbance appears to shift location slightly compared to the TS disturbance (see Figs. 3e and 3f).

Breakdown to turbulence was preceded by the appearance of large amplitude velocity spikes. Far downstream of the location where the spikes were first observed, they became very large $\left(>30 \% U_{e}\right)$ and began to exhibit high-frequency fluctuations, indicating the beginning of breakdown. ${ }^{22}$ As a way of quantifying the spikes, the number of spikes above a chosen threshold of four times the standard deviation $(\sigma)$ of $u^{\prime}$ in each time trace were counted. Figure 6 shows that the positive spikes occur most frequently near the wall, while the negative spikes occur away from the wall and offset in the spanwise direction from the positive spikes. The maximum number of positive and negative spikes from each yz-scan with and without forcing is plotted in Fig. 7. At these locations, the maximum number of positive spikes with no forcing is consistently higher than the maximum number of negative spikes with no forcing, especially for the two downstream locations. The maximum number of both negative and positive spikes is consistently higher with forcing than with no forcing. The increased occurrence of spikes correlates with the higher unsteady disturbance amplitudes with forcing. Unfortunately, since the forcing resulted in higher amplitudes in all three frequency bands, it is not clear whether the increase in spike occurrence is directly related to the TS instability. However, at the first streamwise location $\left(x_{s h}=65\right)$, there was not much difference in the traveling-crossflow and shear-layer instability amplitudes with and without forcing. Since there is an increase in the number of spikes with forcing at this location (particularly the negative spikes), the increase in spikes is at least partially attributable to the increase in amplitude of the TS disturbance. 
The transition location was measured qualitatively using naphthalene flow-visualization for the cases with and without forcing. There was no noticeable change in the transition location between the two cases. While there is an increase in the number of spikes with forcing, breakdown is still intermittent. The increase in spikes caused by the forcing is apparently not enough to cause a visible change in the transition front.

\section{Interaction of TS and Stationary Crossflow}

\section{A. Data Analysis}

It was possible to obtain phase-locked results with the speaker since there was high coherence between the forcing signal and the disturbances in the boundary layer at the forcing frequency. Thus, phase-averaged velocity profiles were computed and were used to observe the interaction of the stationary crossflow and the TS disturbance. This data acquisition and analysis was performed with forcing at $300 \mathrm{~Hz}$ for yz-scans at two streamwise locations (60 and 82 step heights downstream of the step), and streamwise scans (i.e., x-y surfaces) at two locations. The first streamwise scan was performed along a streamline corresponding to the location at which the positive spikes occurred most frequently. The second streamwise scan was performed along a streamline corresponding to the spanwise location of the most negative spikes.

It was necessary to sample the function generator (forcing) signal for these scans in order to perform the data analysis. Phase-averaged data at each point were obtained as follows. The $u^{\prime}$ time trace was shifted by the amount required for the simultaneously acquired forcing signal to be at the same phase as the reference signal (this reference signal was chosen to be the forcing signal from the first point in the first yz-scan acquired). The time signal was then split into segments with length corresponding to the period of the forcing frequency. With a $25 \mathrm{kHz}$ sampling rate, $300 \mathrm{~Hz}$ corresponds to 83.333 time steps. Of course, the length of the segment has to be an integer, so the segments were 83 points long. After every three time segments, a single point was dropped from the time sequence to allow for phase alignment of the next segment. Thus, there may be some minor noise associated with the imperfect phase alignment.

The segments were then averaged to obtain the phase-averaged signal. This was repeated at each point in the boundary-layer scan. It was found that a harmonic of the forcing frequency was present inside the boundary layer. Since the goal was to phase-average the primary mode, the signal was digitally filtered prior to phase-averaging using a band-pass filter centered around the forcing frequency.

\section{B. Results}

Results for the two yz-scans are shown in Figs. 8-16 at equal time intervals throughout the period. For now, the discussion will be limited to the two plots on the top of the figures ( $a$ and $b$ ). The color contours in these plots represent the instantaneous phase-averaged velocity. Note the different color scales in these plots. At the downstream location, the disturbance reaches a higher peak amplitude. Since this disturbance is traveling in the streamwise direction (out of plane), these figures are showing a picture of the distorted TS wave as it passes through this plane. As the TS wave passes through the $\mathrm{y}-\mathrm{z}$ plane, the positive peak occurs off the wall (Fig. 9a,b) and then moves toward the wall (Figs. 10-12a,b). Then the negative peak does the same (Figs. 13-16a,b). Typically, in the absence of stationary crossflow, the TS wave would be characterized by constant bands across the span (i.e., there should be no spanwise variation in amplitude or phase) since the TS wave is traveling close to the streamwise direction. A variation in amplitude of the TS wave across the span is clear in these figures. A variation in phase is particularly evident near the wall $(y \leq 1 \mathrm{~mm})$, but can be seen more readily if we plot the phases across the span at particular $y$-locations. These plots are shown in Fig. 17 for both planes. The results have been unwrapped to eliminate phase jumps larger than $\pi$. Particularly for the upstream location (Fig. 17a), there is a variation of the phase across the span with a wavelength of $11 \mathrm{~mm}$. However, for $z>170 \mathrm{~mm}$, the behavior changes close to the wall. Farther downstream, the $11 \mathrm{~mm}$ variation is not quite as strong, and in fact it appears as though the TS wave may have some component of travel in the spanwise direction, since the lines near the wall have an overall positive slope. Farther from the wall, the $11 \mathrm{~mm}$ variation is still clear. This spanwise variation of the phase illustrates that the wavefront of the TS wave is distorted by the stationary crossflow. Evidently, the stationary crossflow, even at very small amplitudes of approximately $2-3 \% U_{e}$, can cause a distortion of the TS-instability amplitude and wavefront.

Results for the two xy-scans are shown in Figs. 18-26 at equal time intervals throughout the period. The xy-scans were performed by choosing an initial $z$-location at the most upstream x-location, and then 
following the curvature of the streamline downstream, which is approximately the trajectory of the stationary crossflow vortices. Thus, the initial $z$-location $\left(z_{1}\right)$ is denoted for each scan. The $z$-location of the first scan $\left(z_{1}=154.5 \mathrm{~mm}\right)$ was chosen to correspond to the approximate location of maximum positive spikes, while the second one $\left(z_{1}=157.5 \mathrm{~mm}\right)$ was chosen as the approximate location of maximum negative spikes.

There is a $180^{\circ}$ phase shift occurring near the edge of the boundary layer, as would be expected of a TS instability. From these results we can also see that the wave is traveling downstream, with a phase speed of approximately $30 \% U_{e}$, which is in agreement with the previously reported phase speed measurements for the TS instability. ${ }^{5}$ This phase speed is computed by tracking a peak downstream over one cycle, thus computing the speed based on how far the peak traveled. For the first scan (Fig. 18a), the amplitude of the mode increases until approximately $x_{s h}=60$ before starting to decay. In the second scan (Fig. 18b), the amplitude starts to decay earlier, around $x_{s h}=40$. In this scan, the mode appears to split apart starting at around $x_{s h}=60$, resulting in a double-peaked mode shape. This is due to the distortion of the TS instability, and is just starting to become evident in the downstream yz-scan (see Fig. 9b). This double-peaked mode shape behavior was also seen in the computations of Choudhari and Fischer ${ }^{8}$ (see Fig. 9c).

These results illustrate the complex nature of the interaction between the TS and stationary crossflow instabilities. The stationary crossflow causes a distortion of the TS wavefront, which results in a complex flow field, the nature of which may lead to some unknown breakdown mechanisms. The breakdown to turbulence of this type of flow field is not understood and will be examined further in the following sections.

\section{Analysis of Spikes}

As mentioned earlier, large amplitude velocity spikes occur prior to breakdown and appear to be the main mechanism leading to breakdown. In order to understand how transition occurs in this case, we need to understand more about the spike mechanism and what causes it.

\section{A. Data Analysis}

In order to determine the relationship of the large velocity spikes to the TS disturbance, it was necessary to obtain a phase-averaged version of the spikes. However, due to the somewhat random nature of the spikes, it was not a straightforward task to obtain this result. Several different data analysis techniques were attempted, with varying success. As mentioned in the previous section, the time series were split up into sections with length corresponding to the forcing period in order to perform the phase averaging. For the spike analysis, no band-pass filter was applied, and each of these segments was tested to determine if a spike (either positive, negative, or both) had occurred during that segment of time. The criteria for identification of the spikes in this case was 2 standard deviations larger than the entire time series rather than the 4 standard deviations used earlier. This lower threshold was used in order to include more spikes in the analysis, since the spike occurrence is actually rather sparse. Figure 27a illustrates the relationship of the positive spikes to the primary TS mode. This plot is showing the time series split up into longer time segments (three times the period of the $300 \mathrm{~Hz}$ wave) in order to better illustrate how the spikes are related to the primary mode. The spikes here are visible as (mostly positive) large amplitude deviations from the phase-averaged value.

It is clear that the positive spikes occur mainly during the positive part of the primary wave cycle. However, there is visible scatter in the time of arrival, the shape of the spikes, and the amplitude. This is probably due to several factors, primarily the broadband nature of the disturbance field. Even though we are forcing at a single frequency, there is still a lot of energy in a broad range of frequencies. Additionally, the flow mechanism that causes these spikes appears to be convecting downstream. Previous hotwire measurements ${ }^{23}$ were performed in which a fixed wire was placed downstream of the traversing hotwire. The spikes were measured at the downstream station shortly after they occurred at the upstream station, indicating that the spikes were, in fact, convecting downstream. Thus, if spikes at this location are being generated and are convecting from upstream simultaneously, some amount of scatter is to be expected.

Initially, the maximum positive or negative amplitude (for either a positive or negative spike, respectively) at each location and each time within the time segment was used as a measure of the instantaneous spike value. This produced satisfactory results. There was evidence of some spatial coherence of these structures and a clear relationship with the forced TS mode.

A second approach was attempted, which reduced the amount of scatter. For each location, the time 
segments that were identified as having positive or negative spikes were analyzed to determine the time of peak amplitude. In general, although these times could appear to be somewhat random, a plot of the histogram revealed an approximately symmetric and unimodal distribution with a maximum number of spikes occurring within a particular time window (see Fig. 27b). Since the spikes could occur near the beginning or end of the time segment, simply taking an average of the spike times would not give the desired result. The times were wrapped around until a normal distribution was found (i.e., until the peak of the histogram occurred in the middle of the time segment). For example, if there were 100 points in the time segment, and the time segment was divided into 10 bins, the time bin from 1-10 would become 101-110, then the second bin would become 111-120, etc. It was found that minimizing the standard deviation was an easy and effective way of determining the necessary wrapping. Once the wrapping was complete, the average was taken, and this average value was used as the time of arrival of the spike. Then, all of the time segments of the spikes occurring within a specified time window around this time $(+/-5$ time steps) were averaged together to obtain the phase-averaged spike time segment at that location.

\section{B. Results}

The results using the second approach are presented in this paper. Results from this analysis indicate that the spikes are, in fact, temporally associated with the TS mode. The results are plotted in Figs. 8-16 for the yz-scans and Figs. 18-26 for the xy-scans. The positive and negative spike average time series are plotted separately (positive in Figs. c and d, negative in Figs. e and f). The velocity contours of the phase-averaged TS mode are overlaid on these plots for reference. Note that different color scales were used for each plot so that the results would be more visible.

Several observations can be made regarding the nature of the spikes. As was evident in Fig. 27a, the positive spikes appear to be strongly correlated with the positive part of the phase of the TS wave. This is evident in both the yz-scans (Figs. 8-16 c and d) as well as the xy-scans (Figs. 18-26 c and d).

In the yz-scans, the peak amplitude of the positive spike tends to occur offset from the TS peak amplitude to the right and slightly closer to the wall (see Fig. 11c). The maximum negative spike amplitude occurs off of the wall (see Fig. 13e). At the upstream location, the appearance of the negative spikes occurs shortly after the appearance of the maximum positive spike amplitude, but precedes the occurrence of the maximum negative amplitude of the TS wave. Farther downstream, the negative spikes occur at approximately the same time as the positive spikes. The negative spikes appear to spread laterally at the downstream location compared to the upstream location (compare Figs. 13e and 11f). These figures are at different times, but they are approximately the times at which the the maximum negative part of the spike occurs at each location.

The xy-scans shed some more light on this behavior. Remember that the first xy-scan $\left(z_{1}=154.5 \mathrm{~mm}\right)$ was taken at a location corresponding to the maximum occurrence of positive spikes, while the second xyscan $\left(z_{1}=157.5 \mathrm{~mm}\right)$ was taken at the location of the maximum occurrence of negative spikes. Thus, when comparing positive and negative spikes, we should focus on Figs. c and f, since these correspond to the locations of maximum positive and negative spikes, respectively. It becomes apparent that upstream (say at $x_{s h}=60$, where the first yz-scan was acquired), the positive spikes do in fact precede the occurrence of the negative spikes. Here, the maximum positive spike amplitude occurs around $t=7.33 \mathrm{e}-4$ seconds (Fig. 20), whereas the peak negative spike amplitude does not occur until $t \approx 1.8 \mathrm{e}-3$ seconds (Fig. 23). In fact, it appears that closer to the step (approximately 40 step heights downstream), the negative spikes (Fig. 18f) are somewhat correlated with the negative part of the TS wave (Fig. 18b). However, farther downstream (around $x_{s h}=70$ ), the negative spikes become more associated with the positive part of the TS wave. The contours of the spikes also start to stretch out and become more slanted (similar to the behavior of the phase-averaged TS contours). Since the maximum positive spike amplitude occurs near the wall, and the maximum negative spike amplitude occurs off the wall, this stretching results in positive and negative spikes occurring at approximately the same time, starting by around $x_{s h}=85$, which is close to the $\mathrm{x}$-location of the second yz-scan.

These results help to elucidate the structure of the spike mechanism, how it develops downstream, and the relationship of this mechanism to the TS mode. The results show that the appearance of this breakdown mechanism is related to the TS mode, and that the timing of the positive spikes correlates with the positive part of the TS wave cycle. 


\section{Higher-order Spectral Analysis}

\section{A. Data Analysis}

The auto-bicoherence provides a measure of the phase-locking between three frequencies in a single time series such that:

$$
\phi\left(f_{1}\right)+\phi\left(f_{2}\right)=\phi\left(f_{1} \pm f_{2}\right)+\Phi
$$

where $\phi$ is the phase of the associated frequency component, and $\Phi$ is the biphase. Bicoherence is used as a measure of the nonlinear interaction between multiple frequencies. Kim and Powers ${ }^{24}$ showed that the square of the bicoherence, $b^{2}$, is a measure of the fractional power of the wave at $f_{3}=f_{1} \pm f_{2}$ due to the coupling of the waves at $f_{1}$ and $f_{2}$. Similar to coherence, bicoherence is a normalized quantity with values ranging from 0 to 1 . Perfect phase-locking is indicated by a value of $b^{2}=1$, whereas $b^{2}=0$ indicates no phase-locking. An example auto-bicoherence plot is shown in Fig. 28. In this figure, $f_{1}$ and $f_{2}$ are the $\mathrm{x}$ - and $\mathrm{y}$-axes, and $f_{3}$ is either the sum or difference frequency. If two simultaneously acquired time series are available, the cross-bicoherence can be used to determine whether $f_{3}$ is a sum or difference frequency by observing in which quadrant the interaction occurs. The bicoherence estimates in this paper were computed by dividing up each time series into 240 records of 1024 points each.

\section{B. Results}

Previous hotwire results indicated the existence of nonlinear interactions between the different families of unsteady disturbances. ${ }^{23}$ The interaction between the TS and traveling crossflow disturbances is investigated further in this section. Plots of the auto-bicoherence at different locations reveal an interaction at $100 \mathrm{~Hz}$ (traveling crossflow) and $300 \mathrm{~Hz}$ (TS). Figure 28 gives an example of the auto-bicoherence at a single location showing a peak at $(300 \mathrm{~Hz}, 100 \mathrm{~Hz})$. The bicoherence between these modes is strongest near the wall at the spanwise locations of the positive spikes. This is illustrated in Fig. 29, which shows the auto-bicoherence at $100 \mathrm{~Hz}$ and $300 \mathrm{~Hz}$ for both yz-scans. The fact that the location of the strongest bicoherence occurs near the location of the positive spikes suggests that the interaction between these two modes may be responsible for the generation of the spike phenomenon, even though the amplitude of the bicoherence is fairly low. The biphase can be computed as well, and this is shown in Fig. 30 for both yz-scans. The biphase is essentially the phase shift between the two modes and their sum or difference frequency, as given in Eq. 1.

The yz plot of the biphase is interesting in that the pattern looks very similar to the skewness plots for these same scans (shown in Fig. 31). The skewness is plotted as a way of showing the spatial locations of the positive and negative spikes. Values of positive skewness indicate that mainly positive spikes are occurring at that location, and vice versa. Of course, the biphase is meaningless if there is no bicoherence, so the phase is only shown at the locations where the bicoherence is greater than 1E-5. Despite the fairly low values of bicoherence, these phase plots show some coherent structures, essentially indicating that there is a $180^{\circ}$ shift in the biphase between the locations of the negative and positive spikes.

By computing the cross-bicoherence of the previously acquired hotwire data in which flow data were acquired simultaneously from two wires ${ }^{23}$ we find that the interaction between the $100 \mathrm{~Hz}$ and $300 \mathrm{~Hz}$ frequencies occurs in the first quadrant, indicating that it is a summing interaction. Thus, the third interacting component of the triad occurs at $400 \mathrm{~Hz}$. We also can show from these previous measurements that the $400 \mathrm{~Hz}$ component, being the same type of disturbance as the $300 \mathrm{~Hz}$ (TS), behaves similarly to the $300 \mathrm{~Hz}$ component. The phase profiles for these two frequencies are illustrated in Fig. 32 for a single $z$-scan and a single boundary-layer profile, which were taken at locations close to the measurement locations of the two yz-scans. These figures show that the phase profiles of the $300 \mathrm{~Hz}$ and $400 \mathrm{~Hz}$ components are very similar, at least across the yz-plane. The phases are expected to vary in the $\mathrm{x}$-direction, since the two frequencies have two different wavelengths and phase speeds associated with them. Given that the phases across the whole yz-scan are similar, we can assume that $\phi\left(f_{1}=300 \mathrm{~Hz}\right) \approx \phi\left(f_{1}+f_{2}=400 \mathrm{~Hz}\right)$, which means that in Eq. $1, \phi\left(f_{1}+f_{2}\right) \approx \phi\left(f_{1}\right)$. This leaves $\phi\left(f_{2}\right) \approx \Phi$, in other words, the phase of the $100 \mathrm{~Hz}$ mode is approximately equal to the biphase, as shown in Fig. 30.

The biphase contours are shown again in Fig. 33, this time overlaid with the $u_{r m s}^{\prime}$ line contours for the traveling crossflow disturbance band $(f=80$ to $200 \mathrm{~Hz})$. It was noted in the previous work ${ }^{5}$ that all of the disturbance bands undergo distortion due to interaction with the stationary crossflow, but the distortion of the traveling crossflow band is different from the other two. It is known that stationary crossflow can cause a 
distortion of a small-amplitude traveling crossflow wave, examples of which can be seen in Malik et al. ${ }^{25}$ and Li and Choudhari. ${ }^{26}$ Malik et al. ${ }^{25}$ show cases in which two peaks are observed in the rms of the traveling crossflow mode, one close to the wall where the high momentum fluid is pushed down towards the wall, and one (with lower amplitude) farther away from the wall. This is very similar to what is seen in the current experiment (see Fig. 34a and b). When we overlay the $u_{r m s}^{\prime}$ contours on top of the biphase contours, there is a striking similarity. If the above assumptions are true, it appears that the component of the traveling crossflow instability that is off the wall (i.e., centered around $z=152,162$, and $172 \mathrm{~mm}$ ) is $180^{\circ}$ out of phase with the component that is near the wall (i.e., $z=157,168 \mathrm{~mm}$ ).

Given the apparent connection between the locations of the positive and negative spikes and the locations of these peaks in the traveling crossflow rms amplitude, it appears that the spike mechanism may result primarily from the traveling crossflow wave, or from its interaction with the TS wave. It is also noteworthy that the peak in the TS rms amplitude occurs near the same location as the traveling crossflow peak near the wall (see Fig. 34), which is also similar to where the positive spikes occur and where the increase in bicoherence occurs (Fig. 29). This is more evidence to suggest that the interaction of these two instabilities is responsible for the spike phenomenon.

The questions that still remain are: why do the negative and positive spikes only occur in their respective locations, and how do they reach such large amplitudes? One interesting thing to note is that the positive spikes occur near the wall where the high momentum fluid is pulled down by the stationary crossflow, while the negative spikes occur away from the wall where the low momentum fluid is pulled up by the stationary crossflow. The mean flow contours, plotted in Fig. 31 on top of the skewness contours, illustrate this relationship. Thus, these spikes would increase the base flow distortion, whereas, if the spike locations were swapped (i.e., if the negative spikes occurred near the wall where the high momentum fluid was pulled down, and vice versa), this would actually result in a decrease in the flow distortion.

\section{Summary and Concluding Remarks}

The results from this study confirm our current understanding of the types of disturbances that occur downstream of the backward-facing step. The lower frequency disturbances are not sensitive to acoustic forcing, which is expected of traveling crossflow. However, the acoustic forcing was effective at the higher frequencies, which again is consistent with our understanding of these instabilities as TS and shear-layer instabilities. Additionally, the more frequent occurrence of the large-amplitude velocity spikes that lead to breakdown is shown to be related to the increased amplitude of the TS instability. Forcing in the TSinstability frequency band also resulted in increased amplitudes of the traveling crossflow and shear-layer instabilities, implying that nonlinear interactions are occurring between the different instability types.

Phase-locked results were utilized to study the interaction of the TS mode at $300 \mathrm{~Hz}$ with the stationary crossflow. The results show that the small-amplitude stationary crossflow instability causes a drastic distortion of the TS wave front, resulting in a very complex flow field. The location and timing of the positive and negative spikes were also investigated via the phase-locked results. The spikes do appear to be related temporally to the occurrence of the TS wave. In particular, the positive spikes occur at the same time as the positive part of the TS wave and close to the same location, though typically offset in span and a little closer to the wall. The timing and location of the negative spikes vary depending on the streamwise location. Approximately 80 step heights downstream of the step, the positive and negative spikes begin to occur simultaneously. The results from the higher-order spectral analysis indicate that the modulated traveling crossflow disturbance may also be an important player in the generation of this spike breakdown mechanism.

It is interesting that at such small disturbance amplitudes $\left(2-4 \% U_{e}\right)$, these instabilities, in concert with the stationary crossflow, can cause such large velocity fluctuations of approximately 10 times the unsteady rms disturbance amplitude. The big question remaining is how exactly this mechanism occurs. We have shown that the TS and traveling crossflow instabilities both play a role. The stationary crossflow also plays an important role because it causes the spanwise modulation of the unsteady disturbances. Further study is required in order to understand how this breakdown mechanism occurs so that we can better predict the effect of backward-facing steps on transition in swept-wing flows. 


\section{Acknowledgments}

This work was performed as part of the Revolutionary Computational Aerosciences discipline under the Transformational Tools and Technologies Project of NASA's Advance Air Vehicles Program. The authors would like to thank Charlie Debro for his support of the wind tunnel testing and the members of the Flow Physics and Control Branch of NASA Langley Research Center for their support and many helpful discussions.

\section{References}

${ }^{1}$ Perraud, J. and Seraudie, A., "Effects of Steps and Gaps on 2D and 3D Transition," European Congress on Comp. Methods in Applied Science and Eng., ECCOMAS, 2000, pp. 11-14.

${ }^{2}$ Duncan Jr, G., Crawford, B., and Saric, W., "Effects of Step Excrescences on Swept-Wing Transition," AIAA Paper 2013-2412, 2013.

${ }^{3}$ Duncan Jr, G. T., Crawford, B. K., Tufts, M. W., Saric, W. S., and Reed, H. L., "Effects of Step Excrescences on a Swept Wing in a Low-Disturbance Wind Tunnel," AIAA Paper 2014-0910, 2014.

${ }^{4}$ Tufts, M. W., Reed, H. L., Crawford, B. K., Duncan Jr, G. T., and Saric, W. S., "Computational Investigation of Step Excrescence Sensitivity in a Swept-Wing Boundary Layer," AIAA Paper 2015-2775, 2015.

${ }^{5}$ Eppink, J., Wlezien, R., King, R., and Choudhari, M., "The Interaction of Stationary Crossflow and a Backward-facing Step in Swept Boundary-Layer Transition," AIAA Paper 2015-0273, 2015.

${ }^{6}$ Bassom, A. P. and Hall, P., "On the Interaction of Stationary Crossflow Vortices and Tollmien-Schlichting Waves in the Boundary Layer on a Rotating Disc," Proceedings of the Royal Society of London. Series A: Mathematical and Physical Sciences, Vol. 430, No. 1878, 1990, pp. 25-55.

${ }^{7}$ Fransson, J., Brandt, L., Talamelli, A., and Cossu, C., "Experimental and Theoretical Investigation of the Nonmodal Growth of Steady Streaks in a Flat Plate Boundary Layer," Phys. Fluids, Vol. 16, No. 10, 2004, pp. 3627-38.

${ }^{8}$ Choudhari, M. and Fischer, P., "Roughness-Induced Transient Growth," AIAA Paper 2005-4765, 2005.

${ }^{9}$ Wassermann, P. and Kloker, M., "Transition Mechanisms in a Three-Dimensional Boundary-Layer Flow with PressureGradient Changeover," Journal of Fluid Mechanics, Vol. 530, 2005, pp. 265-293.

${ }^{10}$ Saric, W. S. and Reshotko, E., "Review of Flow Quality Issues in Wind Tunnel Testing," AIAA Paper 1998-2613, 1998.

${ }^{11}$ Takagi, S., Saric, W., Radeztsky, R., Spencer, S., and Orr, D., "Effect of Sound and Micron-Sized Roughness on Crossflow Dominated Transition," Bull. Am. Phys. Soc., Vol. 36, 1991, pp. 2630.

${ }^{12}$ Deyhle, H. and Bippes, H., "Disturbance Growth in an Unstable Three-Dimensional Boundary Layer and its Dependence on Environmental Conditions," Journal of Fluid Mechanics, Vol. 316, 1996, pp. 73-113.

${ }^{13}$ Radeztsky, R., Reibert, M., and Saric, W., "Effect of Isolated Micron-Sized Roughness on Transition in Swept-Wing Flows," AIAA Journal, Vol. 37, No. 11, 1999, pp. 1370-1377.

${ }^{14}$ Leehey, P. and Shapiro, P., "Leading Edge Effect in Laminar Boundary Layer Excitation by Sound," Laminar-Turbulent Transition, Vol. 1, 1980, pp. 321-331.

${ }^{15}$ Wlezien, R., Parekh, D., and Island, T., "Measurement of Acoustic Receptivity at Leading Edges and Porous Strips," Applied Mechanics Reviews, Vol. 43, 1990, pp. 167-174.

${ }^{16}$ Saric, W. and White, E., "Influence of High-Amplitude Noise on Boundary-Layer Transition to Turbulence," AIAA Paper 98-2645, 1998.

${ }^{17}$ Reed, H. and Saric, W., "Transition Mechanisms for Transport Aircraft," AIAA Paper 2008-3743, 2008.

${ }^{18}$ Tam, C. K., "Excitation of Instability Waves in a Two-Dimensional Shear Layer by Sound," Journal of Fluid Mechanics, Vol. 89, No. 02, 1978, pp. 357-371.

${ }^{19}$ Ahuja, K., Lepicovsky, J., Tam, C., Morris, P., and Burrin, R., "Tone-Excited Jet: Theory and Experiments," 1982.

${ }^{20}$ Goldstein, M., "The Coupling Between Flow Instabilities and Incident Disturbances at a Leading Edge," Journal of Fluid Mechanics, Vol. 104, 1981, pp. 217-246.

${ }^{21}$ King, R. A. and Breuer, K. S., "Acoustic Receptivity and Evolution of Two-Dimensional and Oblique Disturbances in a Blasius Boundary Layer," Journal of Fluid Mechanics, Vol. 432, 2001, pp. 69-90.

${ }^{22}$ Eppink, J. L., Wlezien, R. W., King, R. A., and Choudhari, M., "The Effect of Backward-facing Step Height on Instability Growth and Breakdown in Swept Wing Boundary-Layer Transition," AIAA Paper 2015-0273, 2015.

${ }^{23}$ Eppink, J., The Interaction of Crossflow Instabilities and a Backward-Facing Step in Swept Boundary Layer Transition, Ph.D. thesis, Tufts University, 2014.

${ }^{24}$ Kim, Y. and Powers, E., "Digital Bispectral Analysis and its Applications to Nonlinear Wave Interactions," IEEE Transactions on Plasma Science, Vol. 7, No. 2, 1979, pp. 120-131.

${ }^{25}$ Malik, M., Li, F., and Chang, C.-L., "Crossflow Disturbances in Three-Dimensional Boundary Layers:Nonlinear Development, Wave Interaction and Secondary Instability," Journal of Fluid Mechanics, Vol. 268, No. 1, 1994, pp. 1-36.

${ }^{26} \mathrm{Li}$, F. and Choudhari, M., "Spatially Developing Secondary Instabilities and Attachment Line Instability in Supersonic Boundary Layers," AIAA Paper 2008-590, 2008. 


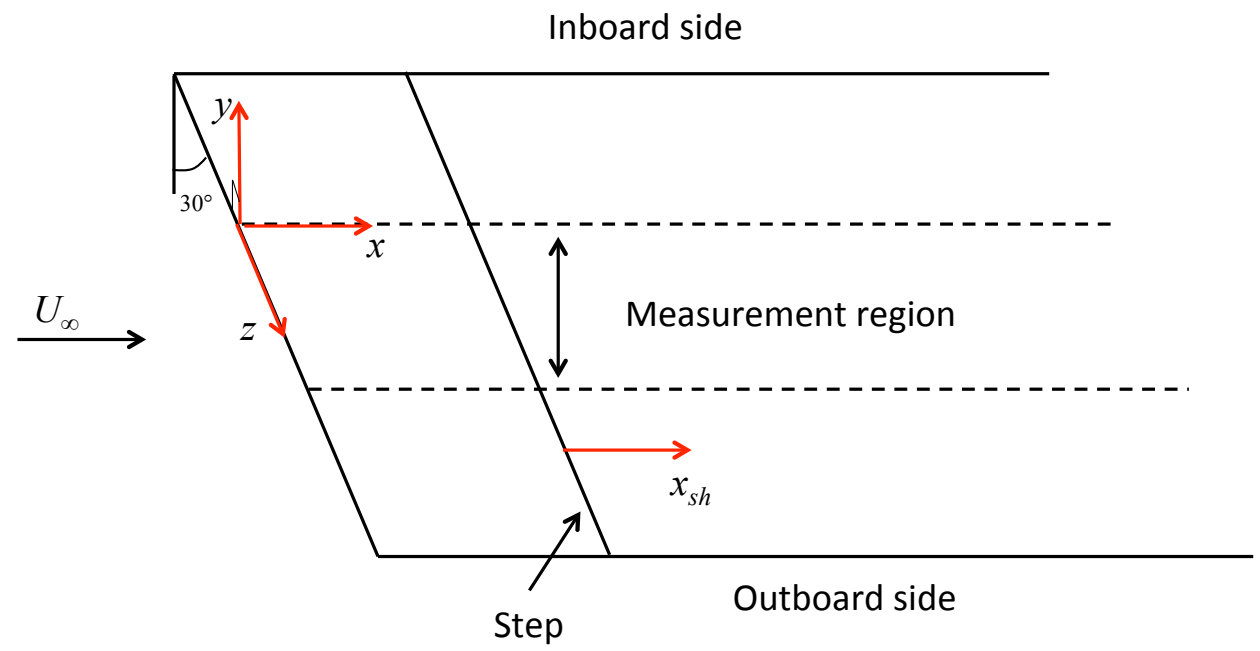

Figure 1: Coordinate system

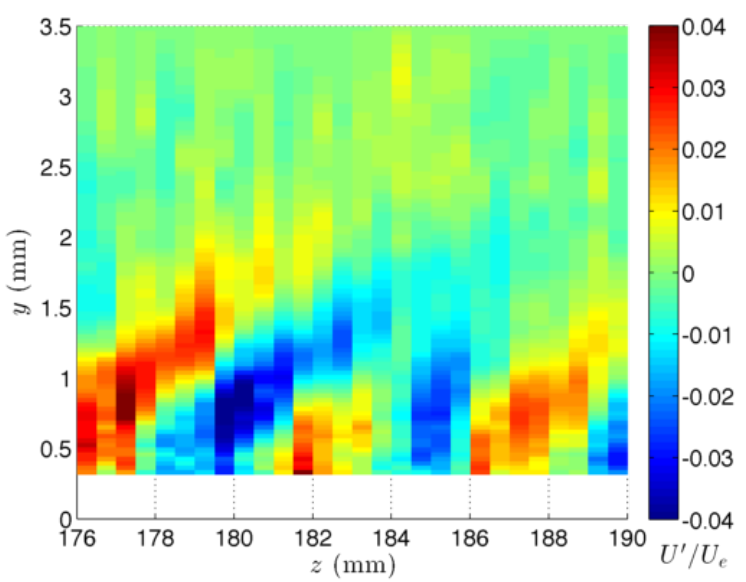

(a) No forcing

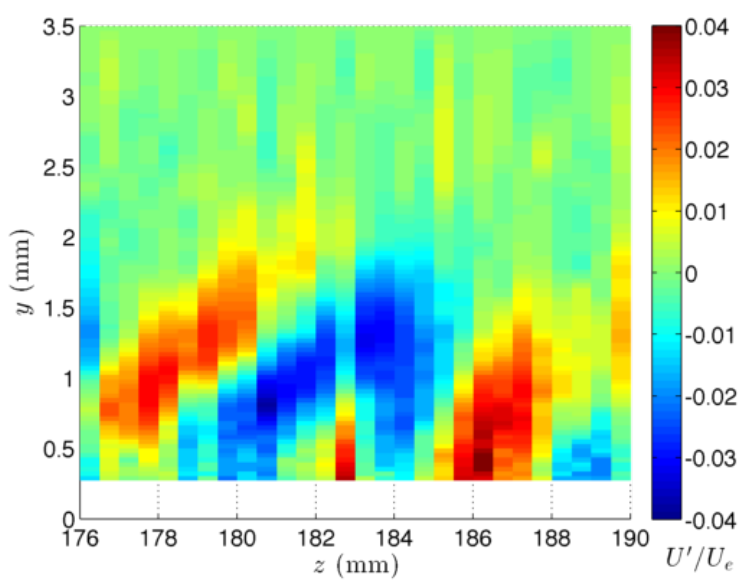

(b) With forcing

Figure 2: Steady disturbance velocity contours at $x_{s h}=100$ 


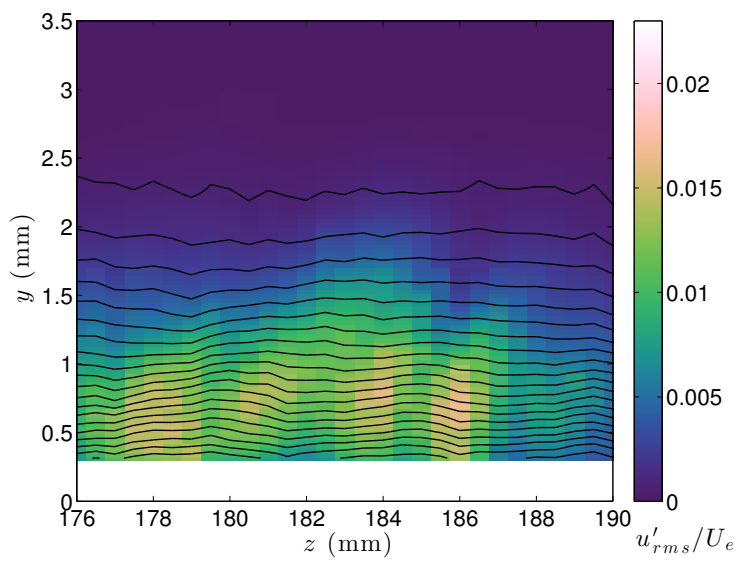

(a) Traveling-crossflow instability, no forcing

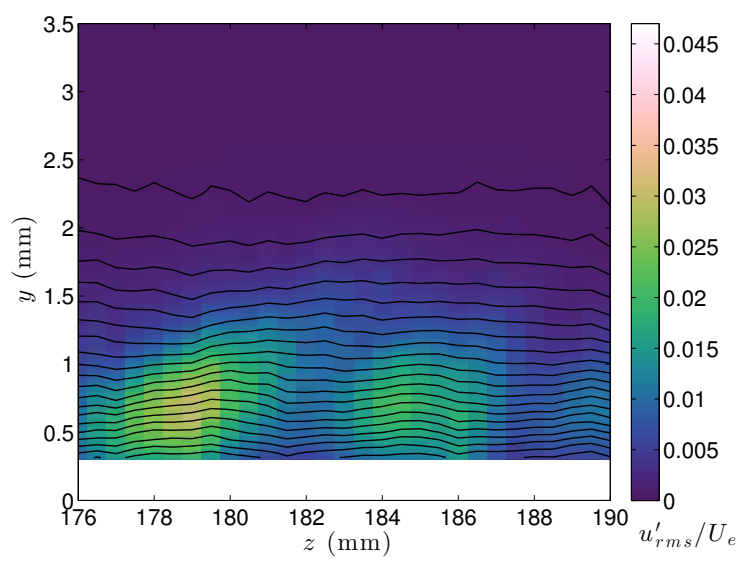

(c) TS instability, no forcing

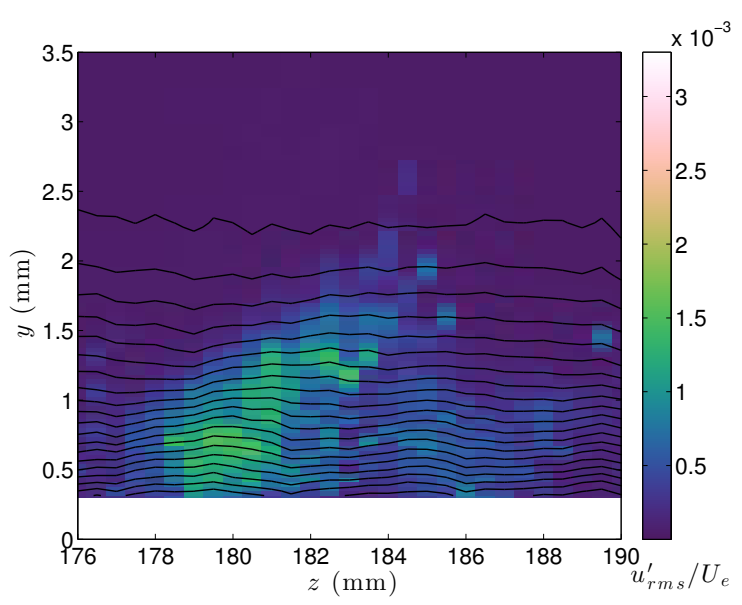

(e) Shear-layer instability, no forcing

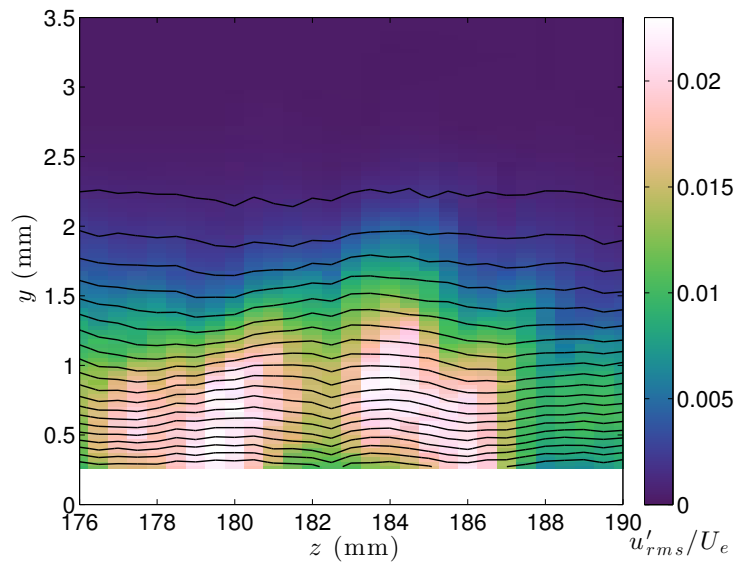

(b) Traveling-crossflow instability, with forcing

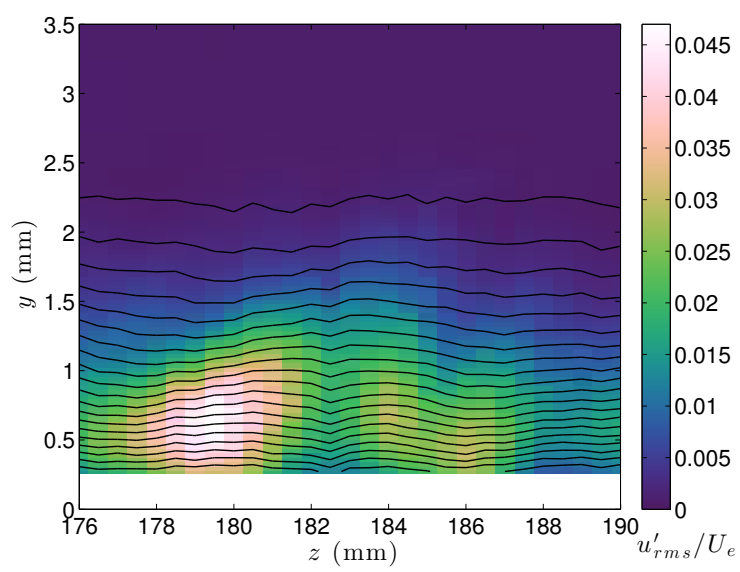

(d) TS instability, with forcing

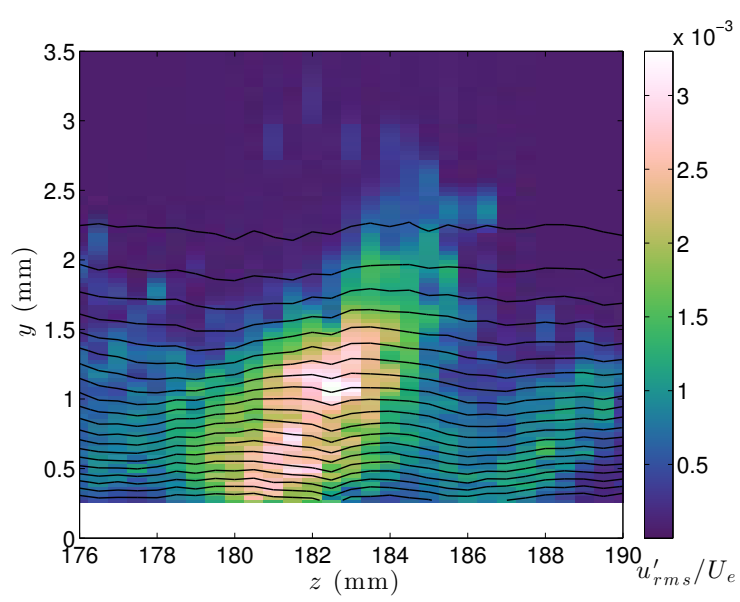

(f) Shear-layer instability, with forcing

Figure 3: Comparison of all disturbance bands $u_{r m s}^{\prime}$ (colors) overlaid with mean-flow contours (lines) with and without $\mathrm{f}=260 \mathrm{~Hz}$ forcing (all measurements at $x_{s h}=100$ ) 


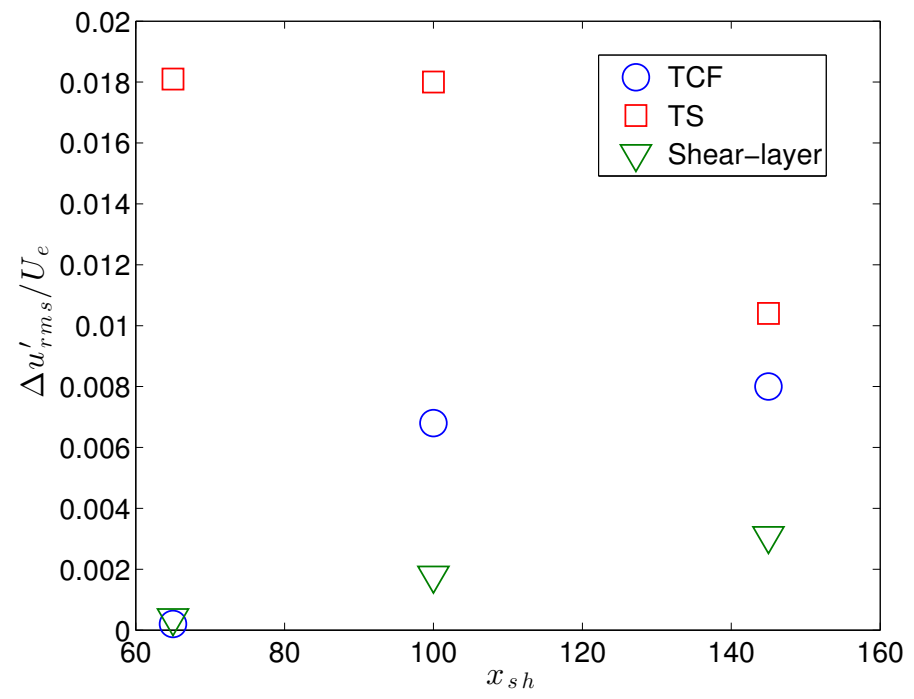

Figure 4: Difference in peak $u_{r m s}^{\prime}$ amplitudes in each band between the forced case $(f=260 \mathrm{~Hz})$ and the unforced case: $\Delta u_{r m s}^{\prime}=u_{r m s, \text { forced }}^{\prime}-u_{r m s, \text { unforced }}^{\prime}$

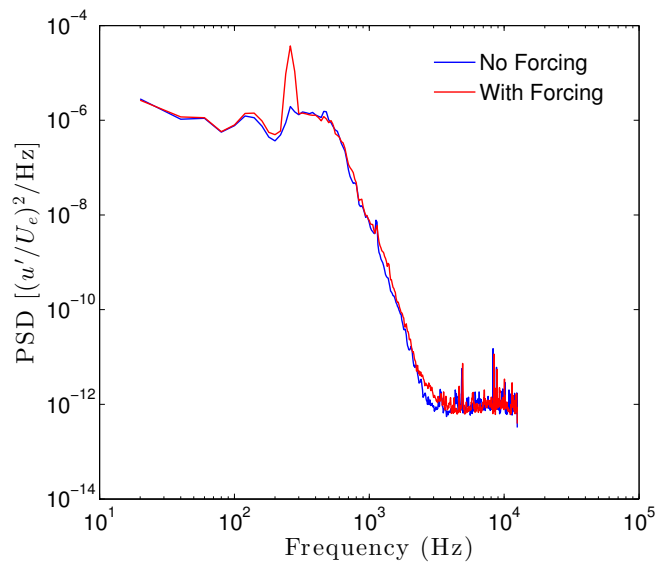

(a) $x_{s h}=65, y=0.8 \mathrm{~mm}, z=177.5 \mathrm{~mm}$

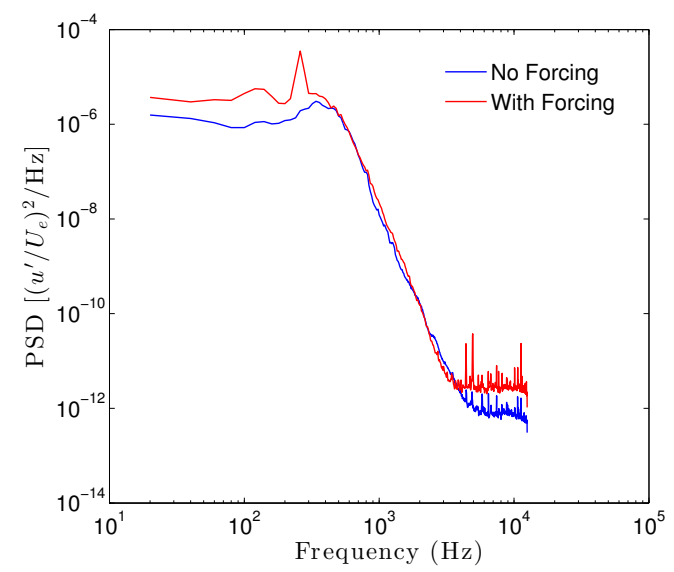

(b) $x_{s h}=100, y=0.8 \mathrm{~mm}, z=179.5 \mathrm{~mm}$

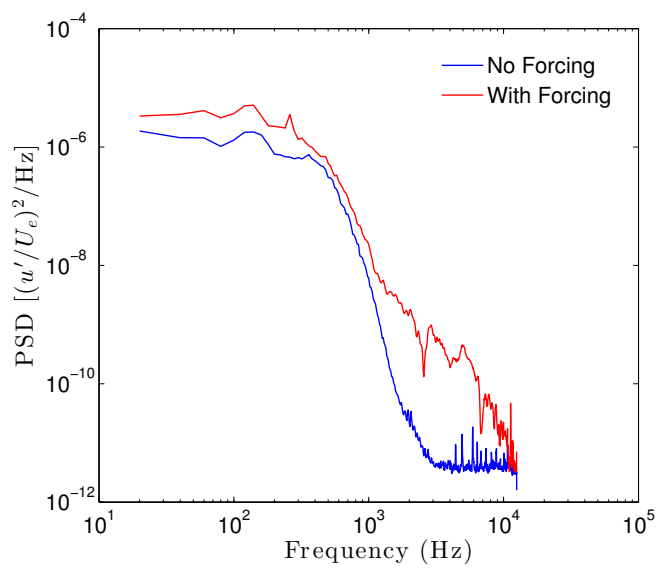

(c) $x_{s h}=145, y=0.8 \mathrm{~mm}, z=181.5 \mathrm{~mm}$

Figure 5: Velocity spectra at similar locations with and without $\mathrm{f}=260 \mathrm{~Hz}$ forcing

\section{4 of 38}




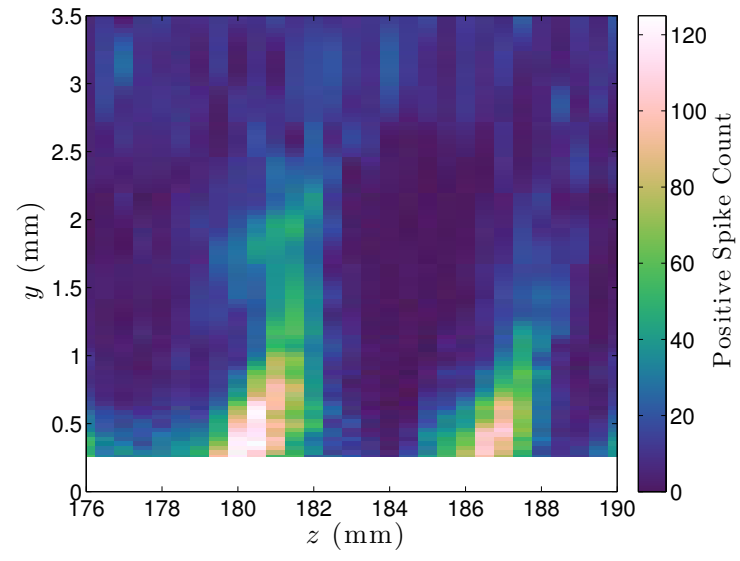

(a) Positive spikes

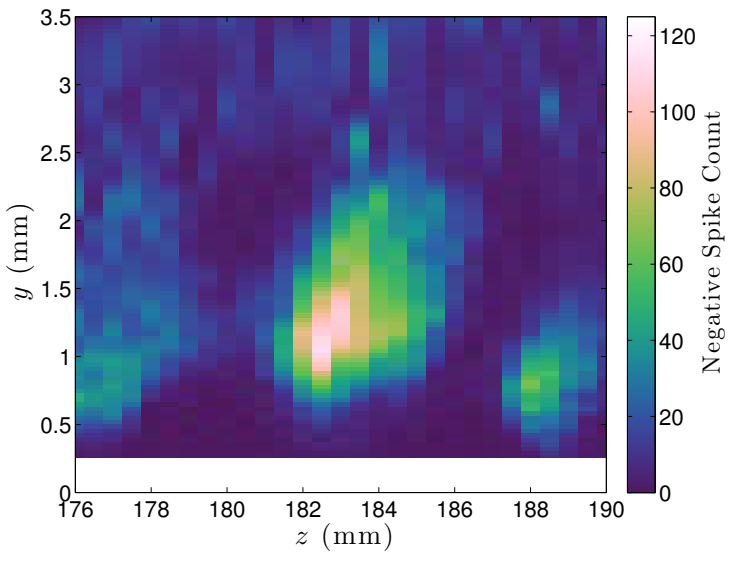

(b) Negative spikes

Figure 6: Spike counts at $x_{s h}=100$ with $\mathrm{f}=260 \mathrm{~Hz}$ forcing

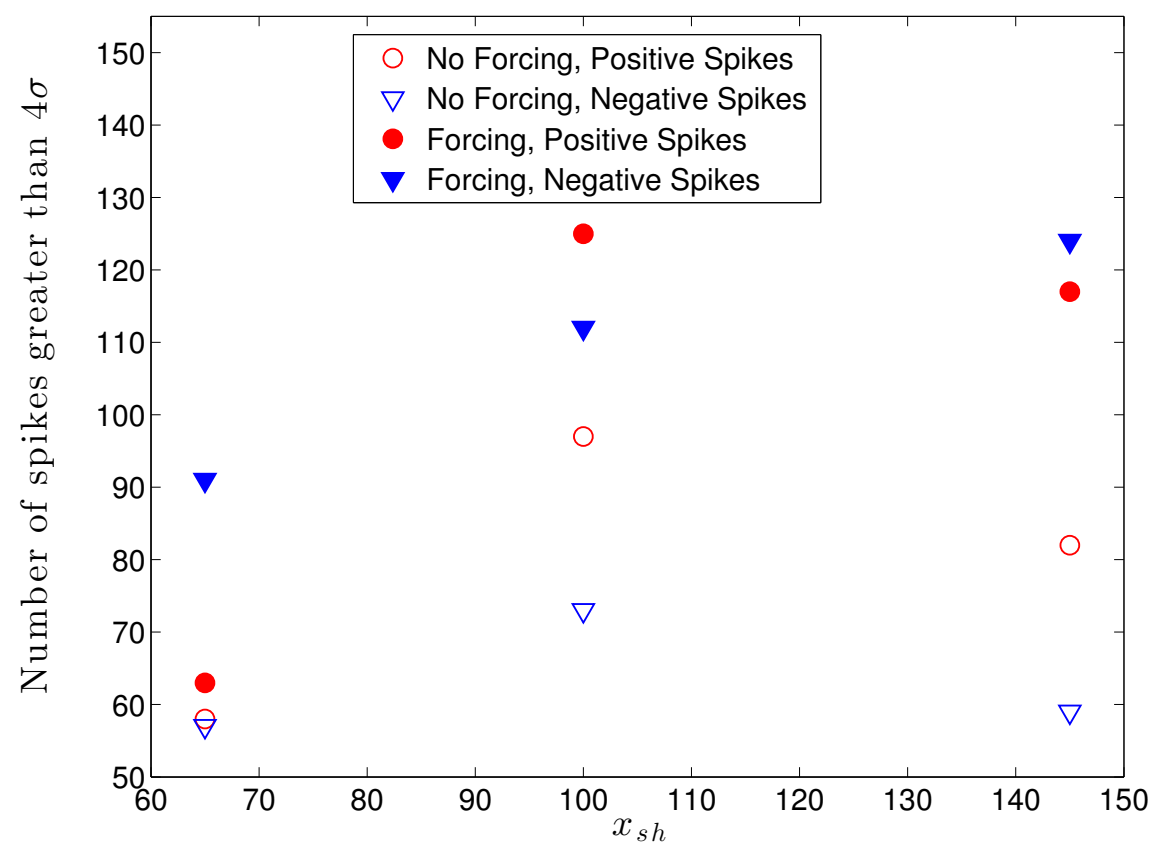

Figure 7: Comparison of positive and negative spike counts with and without forcing 


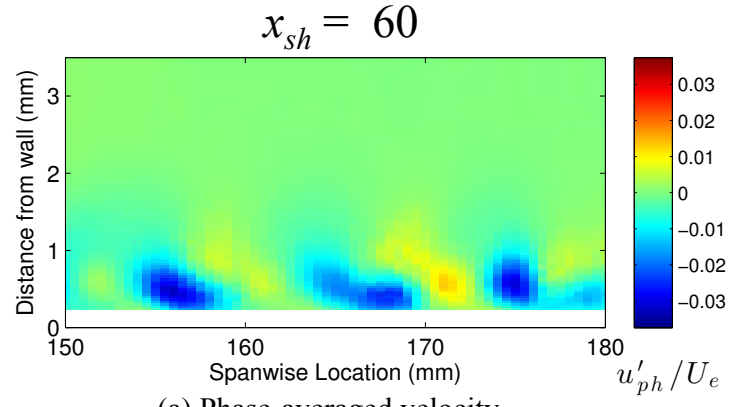

(a) Phase-averaged velocity

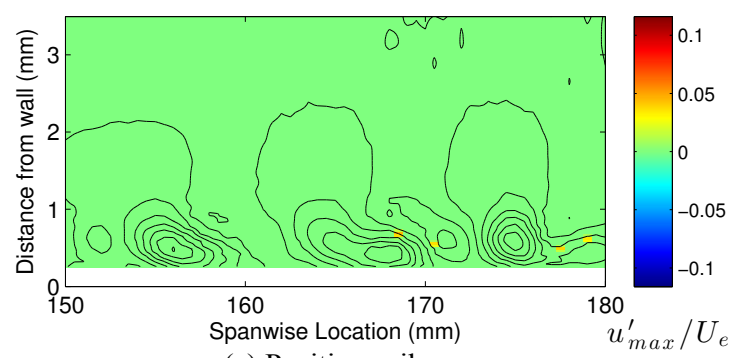

(c) Positive spikes

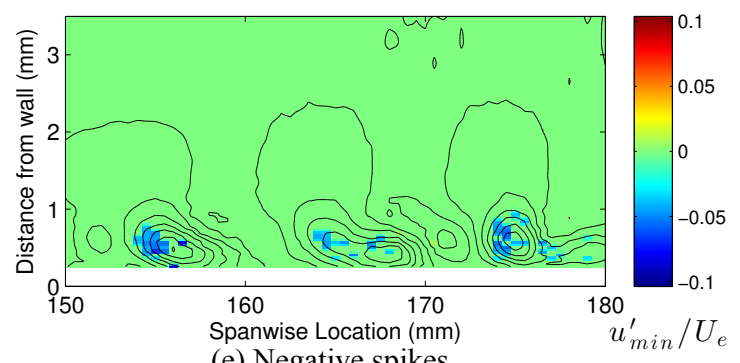

(e) Negative spikes

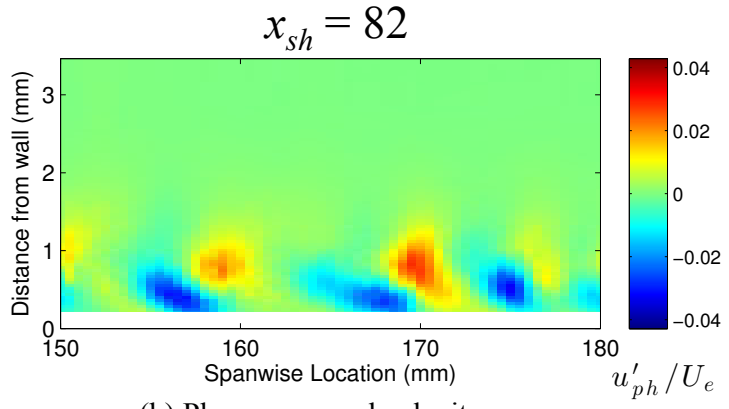

(b) Phase-averaged velocity

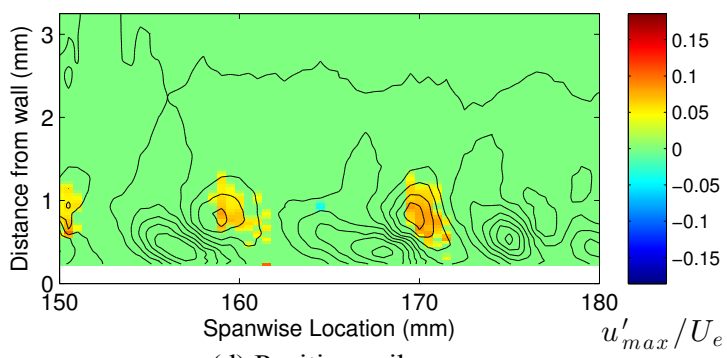

(d) Positive spikes

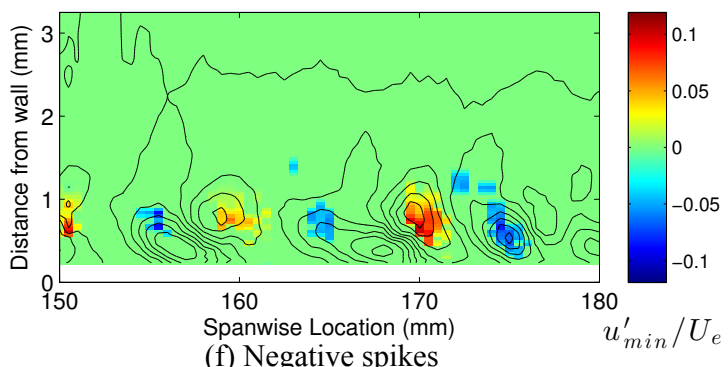

(f) Negative spikes

Figure 8: Phase-averaged yz-scan results with $300 \mathrm{~Hz}$ forcing, $t=0 \mathrm{~s}$. Contour lines of the phase-averaged velocity at the same location are overlaid on Figs. c-f 


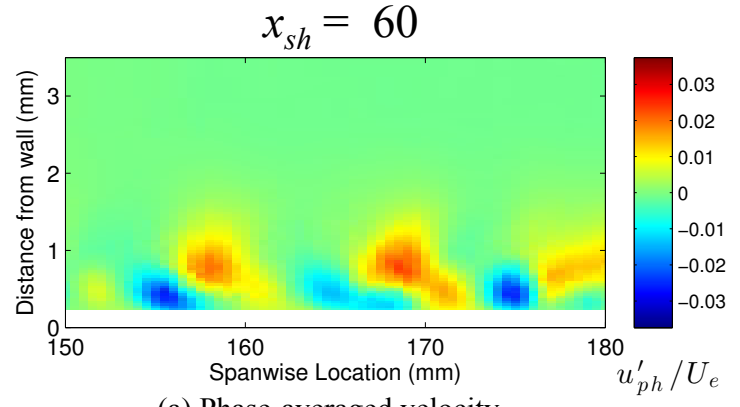

(a) Phase-averaged velocity

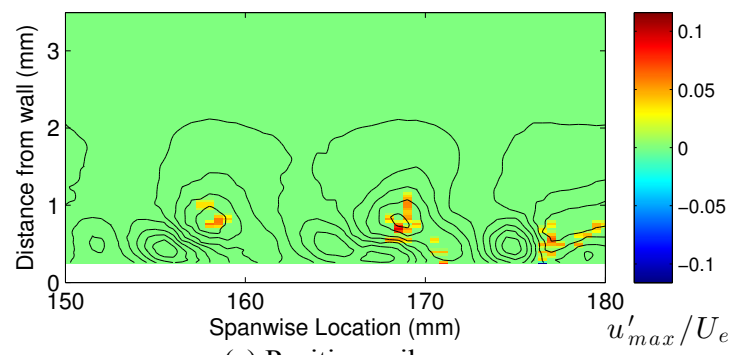

(c) Positive spikes

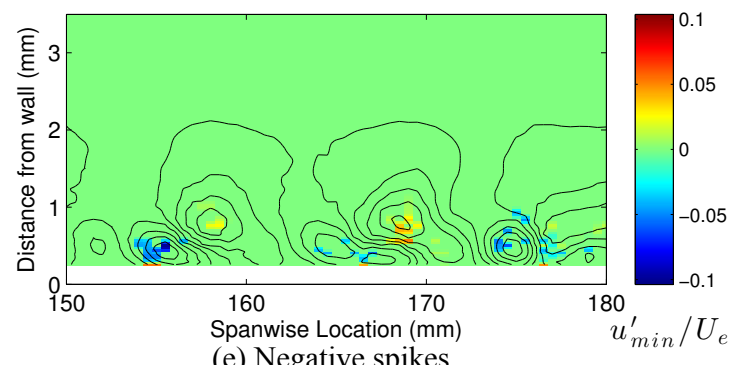

(e) Negative spikes

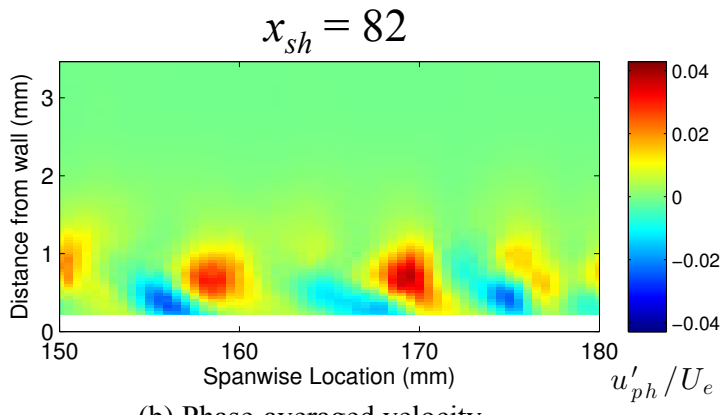

(b) Phase-averaged velocity

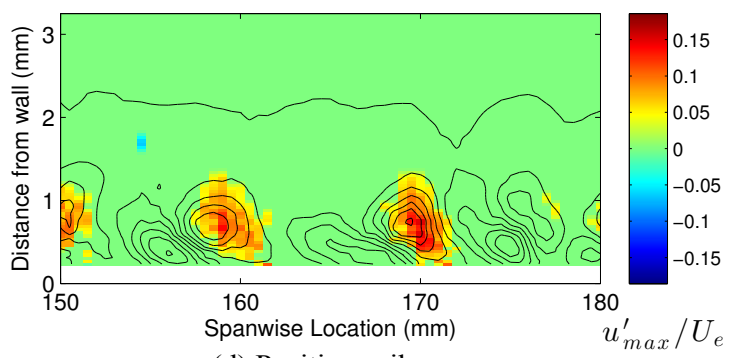

(d) Positive spikes

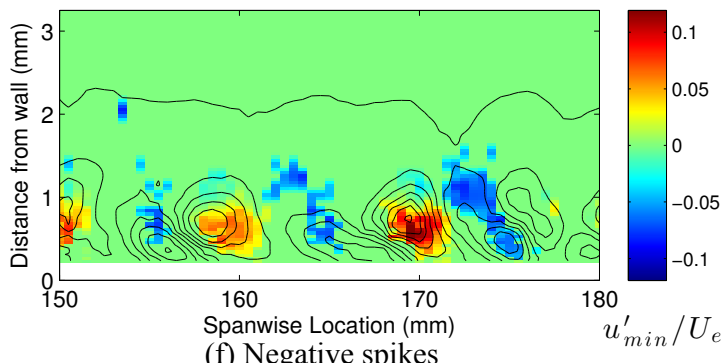

(f) Negative spikes

Figure 9: Phase-averaged yz-scan results with $300 \mathrm{~Hz}$ forcing, $t=3.67 \mathrm{e}-4 \mathrm{~s}$. Contour lines of the phaseaveraged velocity at the same location are overlaid on Figs. c-f 


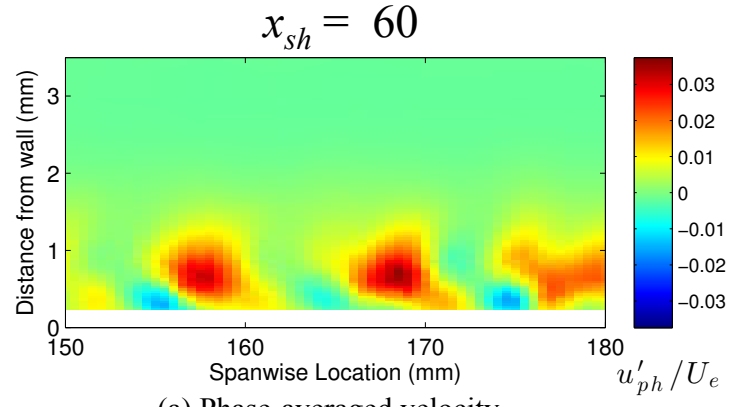

(a) Phase-averaged velocity

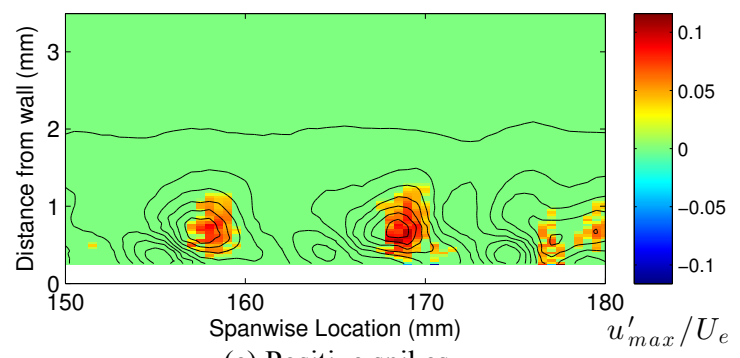

(c) Positive spikes

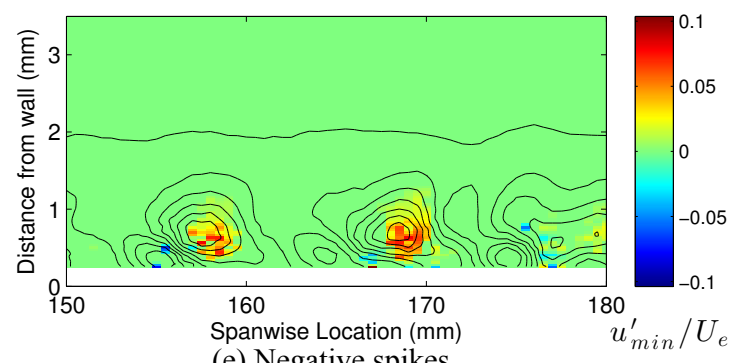

(e) Negative spikes

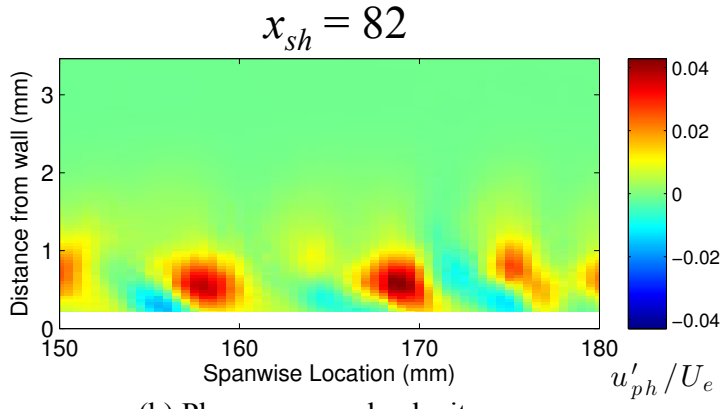

(b) Phase-averaged velocity

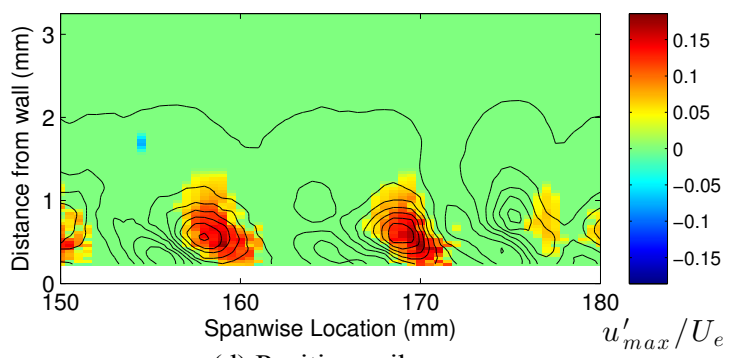

(d) Positive spikes

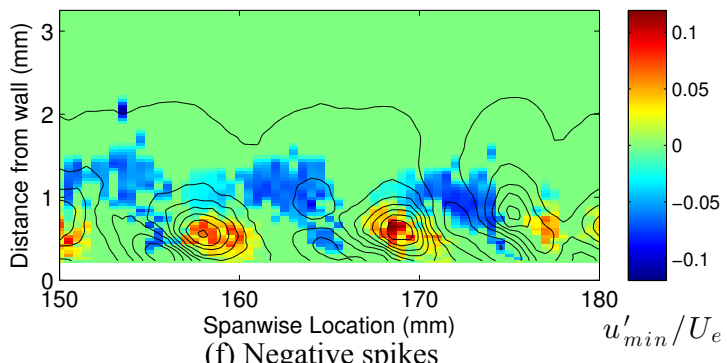

(f) Negative spikes

Figure 10: Phase-averaged yz-scan results with $300 \mathrm{~Hz}$ forcing, $t=7.33 \mathrm{e}-4 \mathrm{~s}$. Contour lines of the phaseaveraged velocity at the same location are overlaid on Figs. c-f 


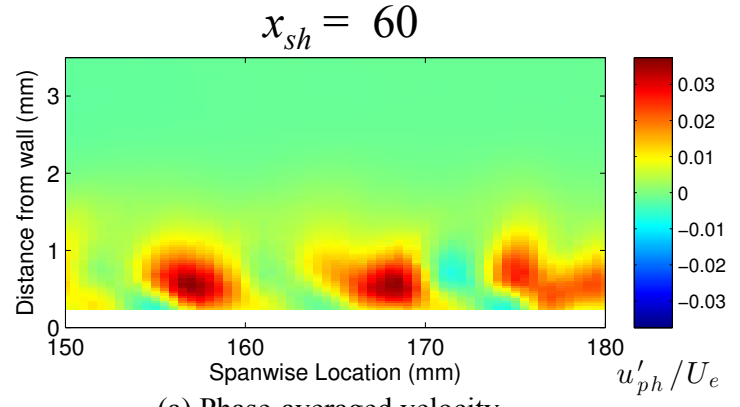

(a) Phase-averaged velocity

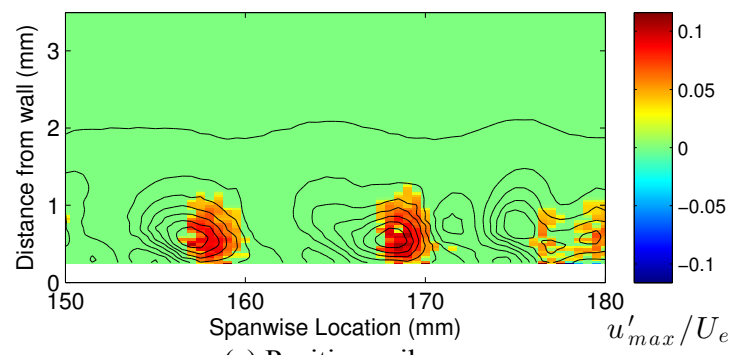

(c) Positive spikes

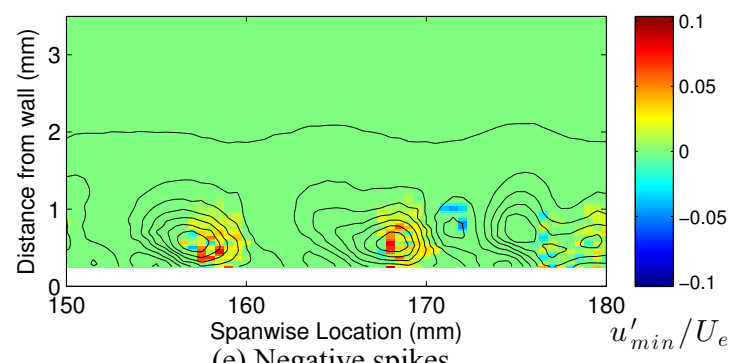

(e) Negative spikes

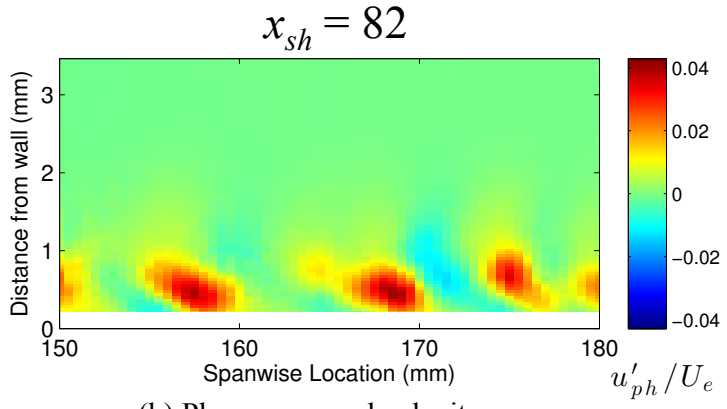

(b) Phase-averaged velocity

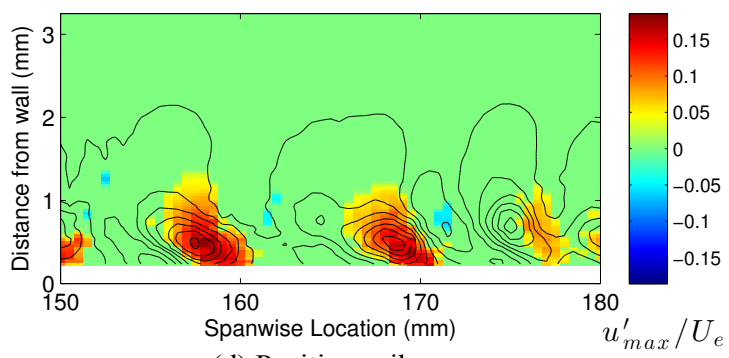

(d) Positive spikes

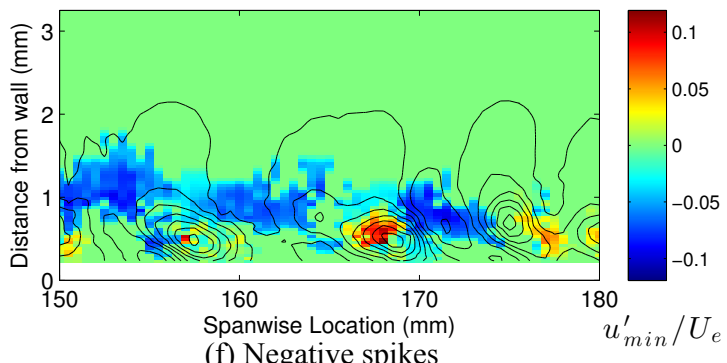

(f) Negative spikes

Figure 11: Phase-averaged yz-scan results with $300 \mathrm{~Hz}$ forcing, $t=1.1 \mathrm{e}-3 \mathrm{~s}$. Contour lines of the phaseaveraged velocity at the same location are overlaid on Figs. c-f 


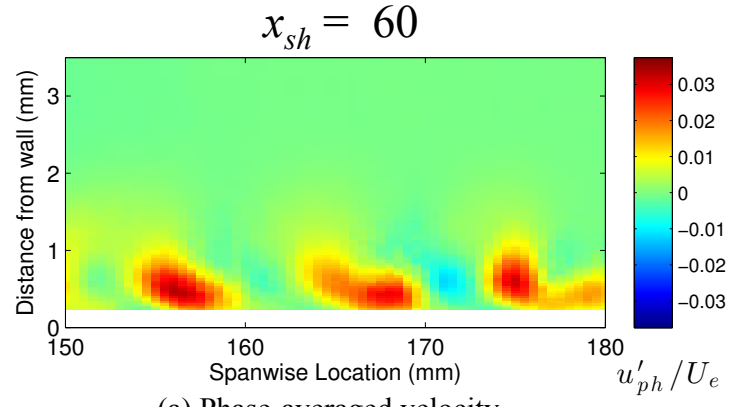

(a) Phase-averaged velocity

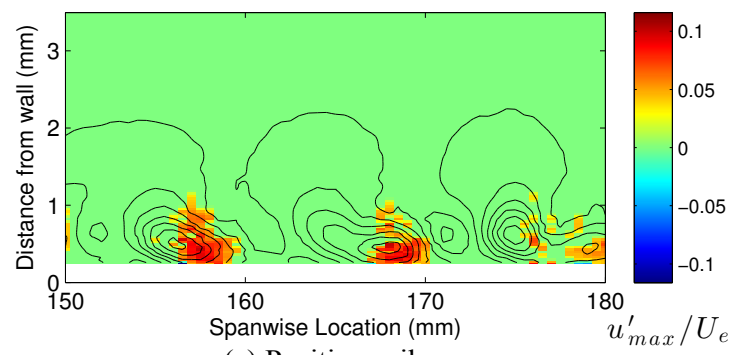

(c) Positive spikes

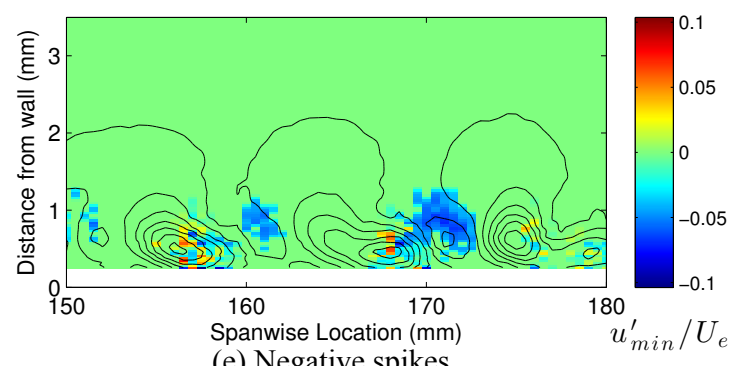

(e) Negative spikes

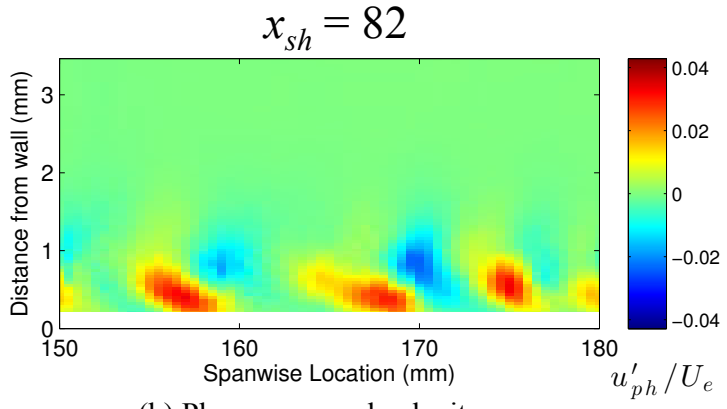

(b) Phase-averaged velocity

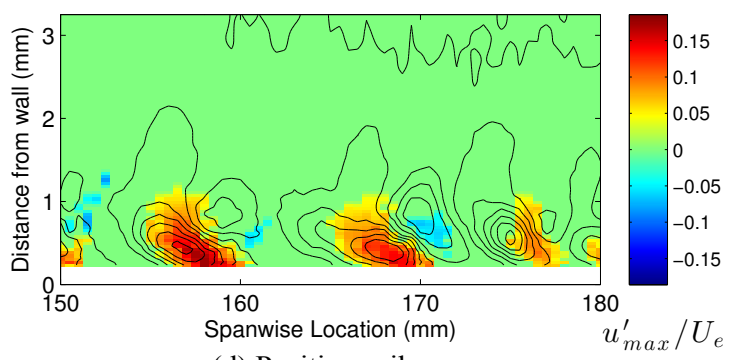

(d) Positive spikes

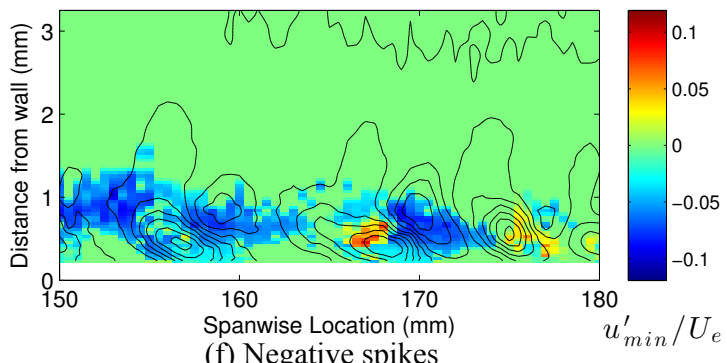

(f) Negative spikes

Figure 12: Phase-averaged yz-scan results with $300 \mathrm{~Hz}$ forcing, $t=1.5 \mathrm{e}-3 \mathrm{~s}$. Contour lines of the phaseaveraged velocity at the same location are overlaid on Figs. c-f 


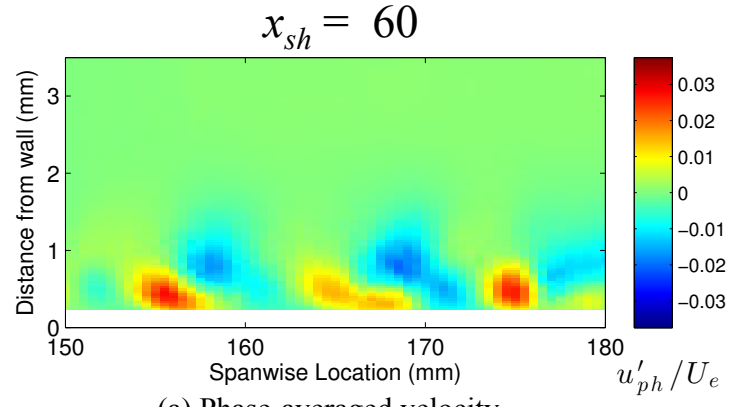

(a) Phase-averaged velocity

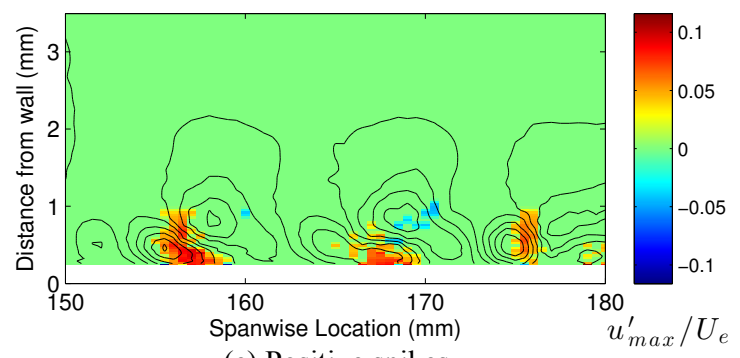

(c) Positive spikes

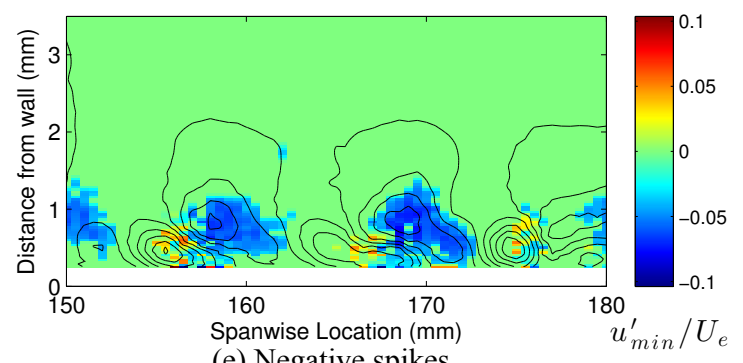

(e) Negative spikes

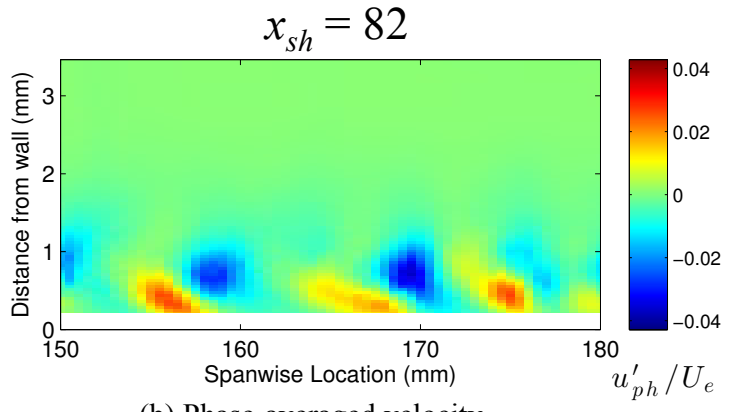

(b) Phase-averaged velocity

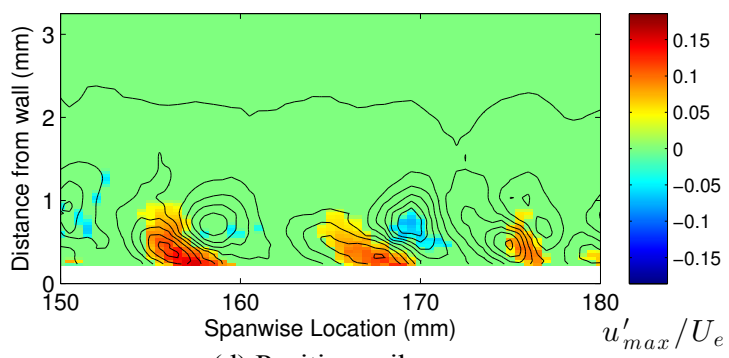

(d) Positive spikes

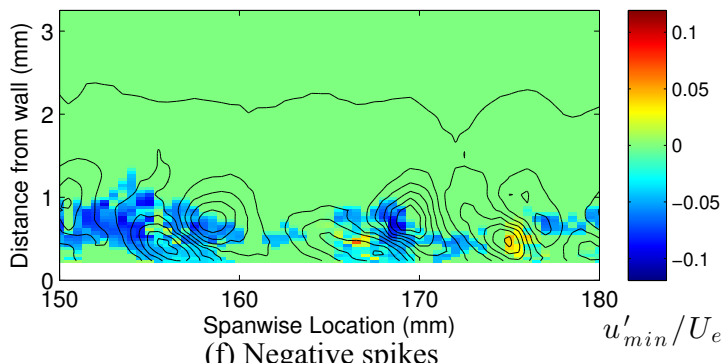

(f) Negative spikes

Figure 13: Phase-averaged yz-scan results with $300 \mathrm{~Hz}$ forcing, $t=1.8 \mathrm{e}-3 \mathrm{~s}$. Contour lines of the phaseaveraged velocity at the same location are overlaid on Figs. c-f 


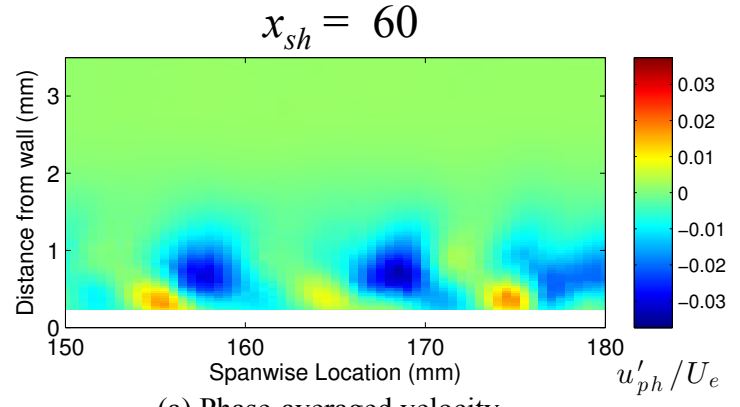

(a) Phase-averaged velocity

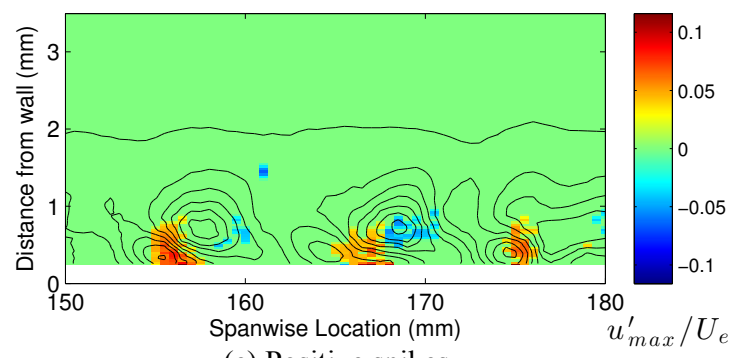

(c) Positive spikes

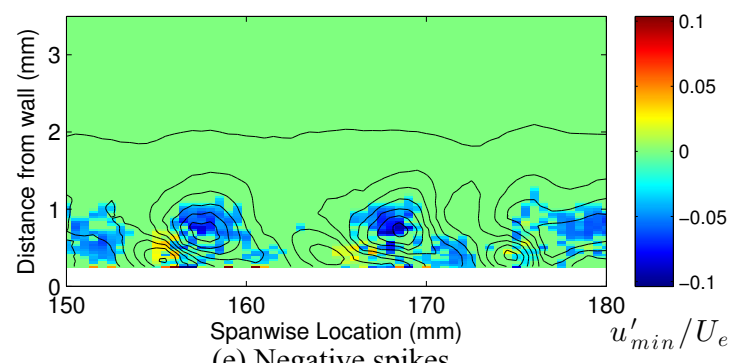

(e) Negative spikes

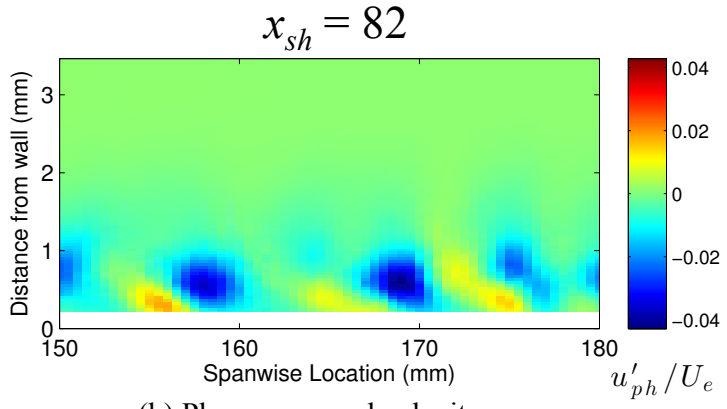

(b) Phase-averaged velocity

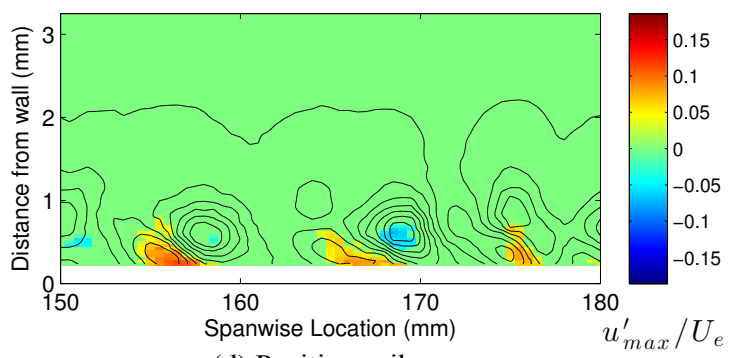

(d) Positive spikes

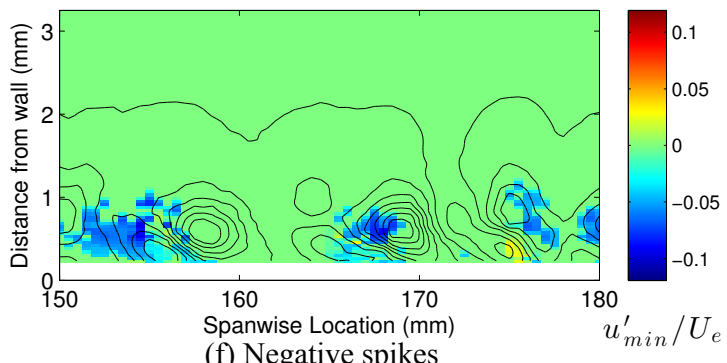

(f) Negative spikes

Figure 14: Phase-averaged yz-scan results with $300 \mathrm{~Hz}$ forcing, $t=2.2 \mathrm{e}-3 \mathrm{~s}$. Contour lines of the phaseaveraged velocity at the same location are overlaid on Figs. c-f 


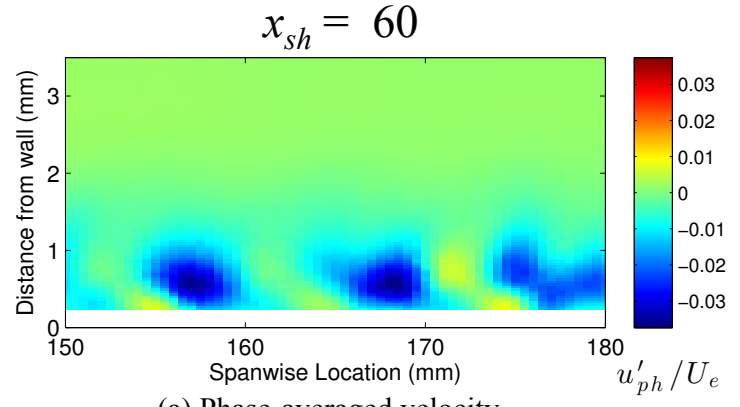

(a) Phase-averaged velocity

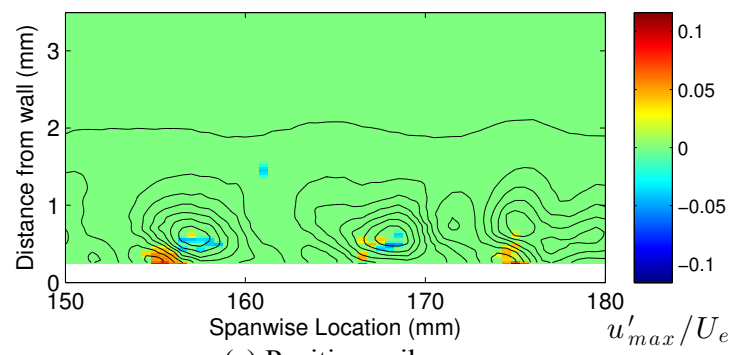

(c) Positive spikes

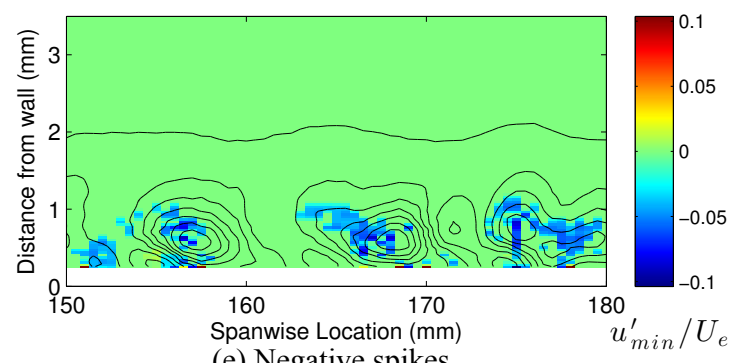

(e) Negative spikes

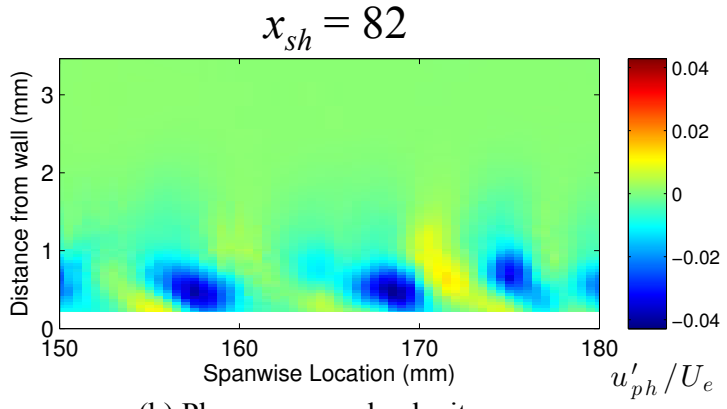

(b) Phase-averaged velocity

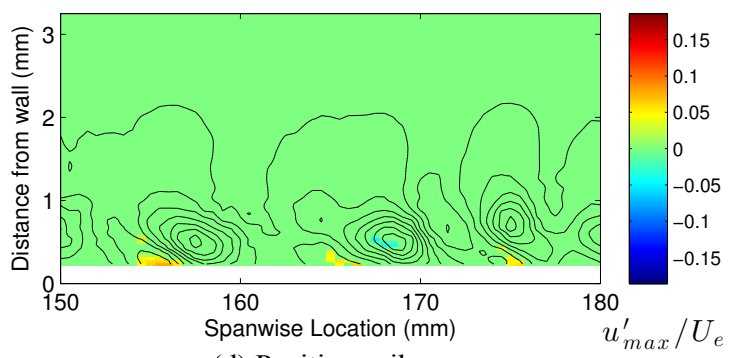

(d) Positive spikes

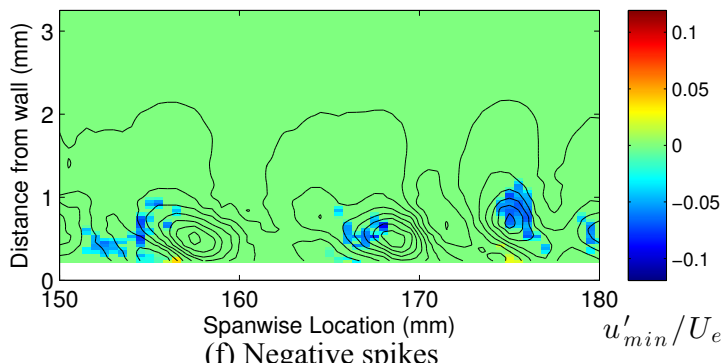

(f) Negative spikes

Figure 15: Phase-averaged yz-scan results with $300 \mathrm{~Hz}$ forcing, $t=2.6 \mathrm{e}-3 \mathrm{~s}$. Contour lines of the phaseaveraged velocity at the same location are overlaid on Figs. c-f 


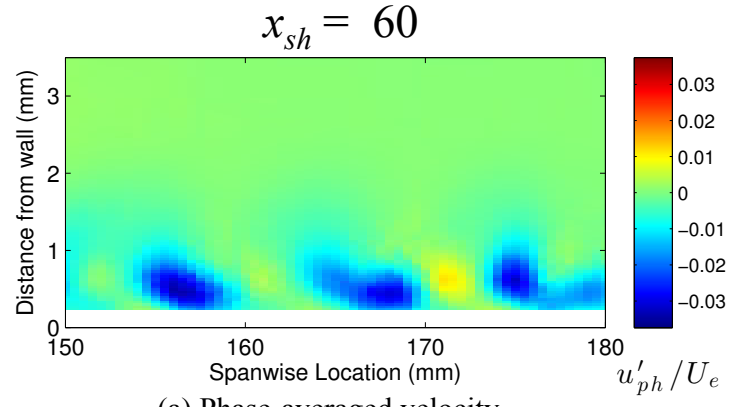

(a) Phase-averaged velocity

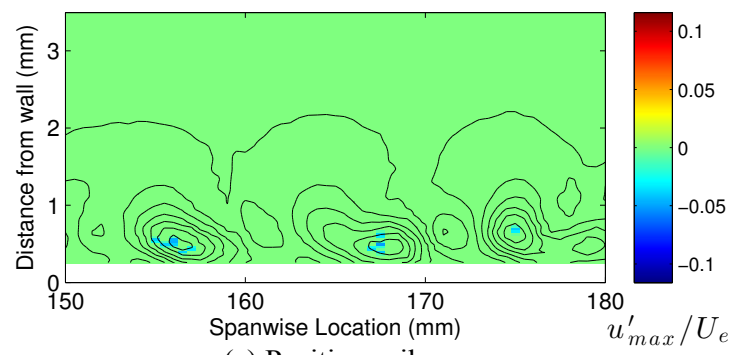

(c) Positive spikes

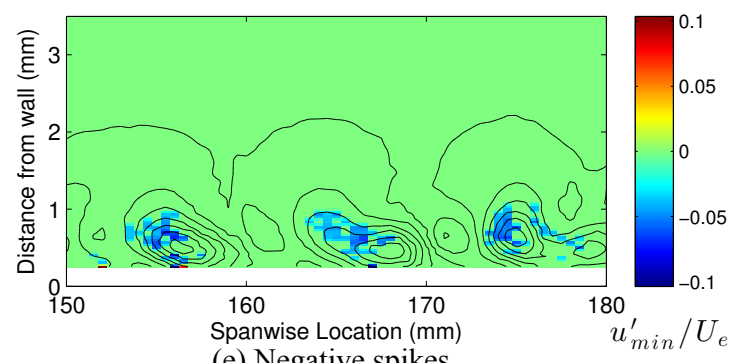

(e) Negative spikes

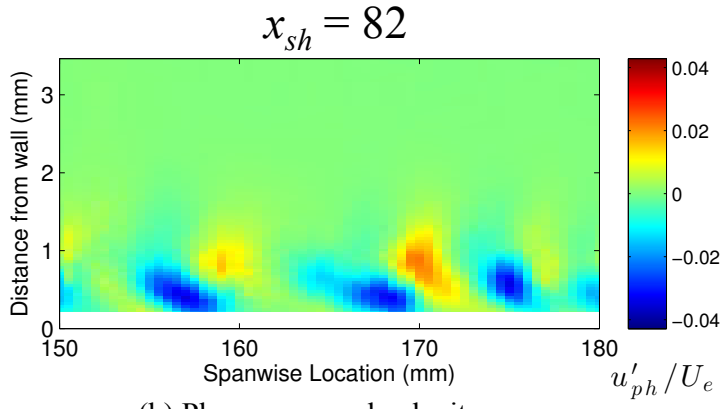

(b) Phase-averaged velocity

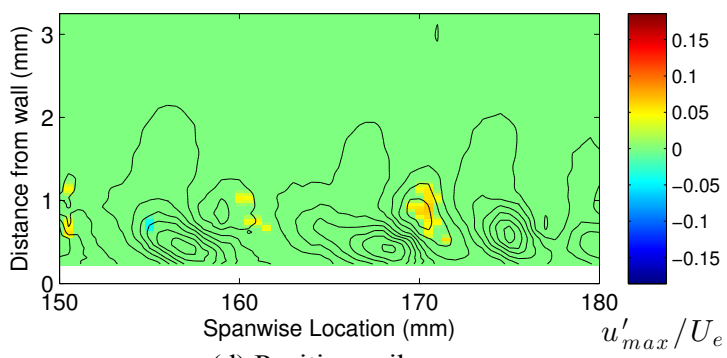

(d) Positive spikes

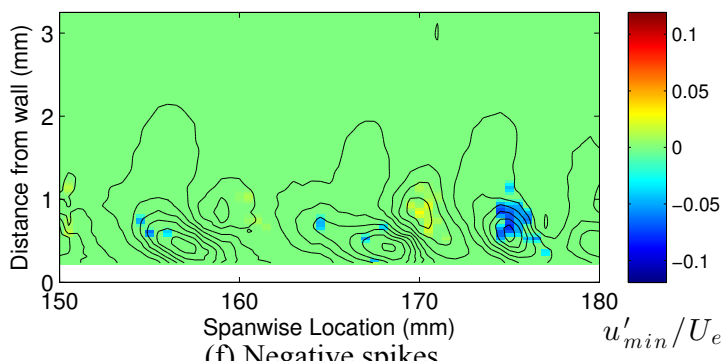

(f) Negative spikes

Figure 16: Phase-averaged yz-scan results with $300 \mathrm{~Hz}$ forcing, $t=2.9 \mathrm{e}-3 \mathrm{~s}$. Contour lines of the phaseaveraged velocity at the same location are overlaid on Figs. c-f 


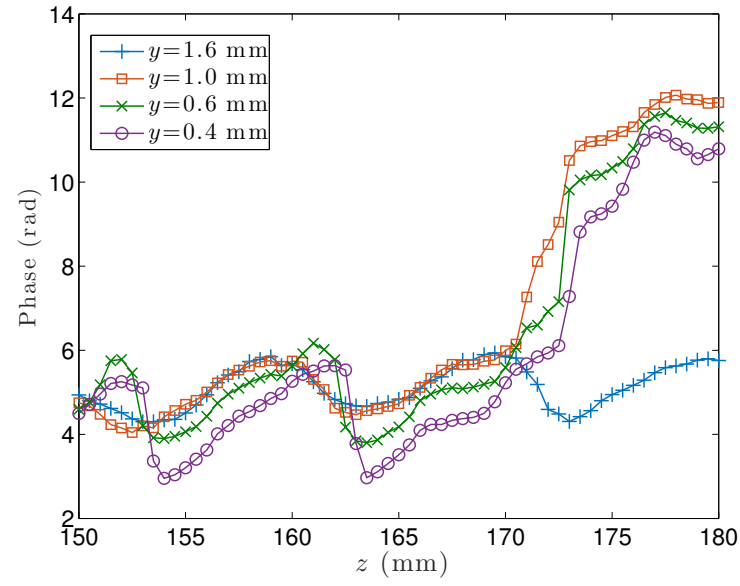

(a) $x_{s h}=60$

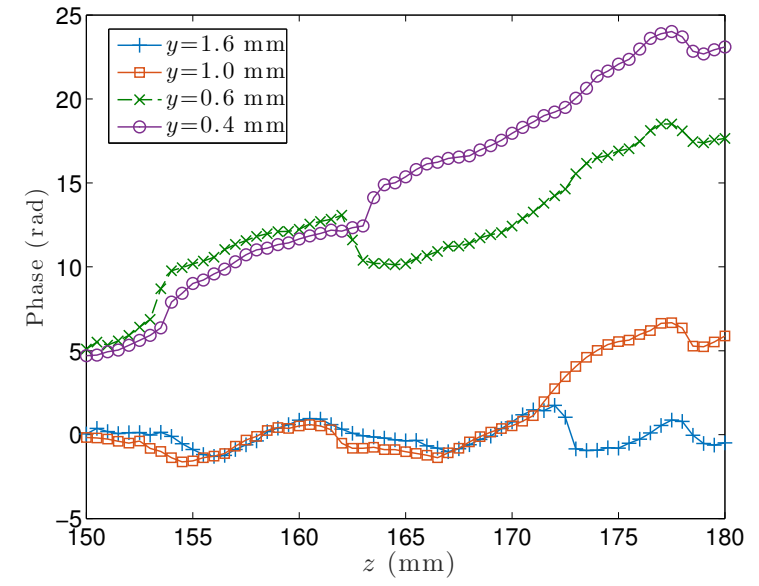

(b) $x_{s h}=82$

Figure 17: Phase of $300 \mathrm{~Hz}$ mode vs $z$ at several $y$-locations 


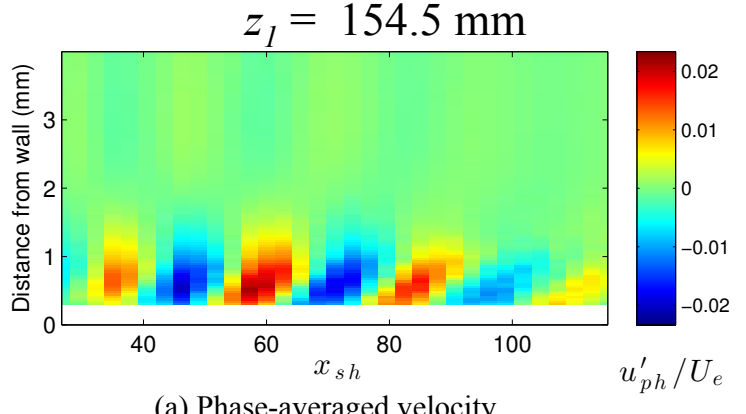

(a) Phase-averaged velocity

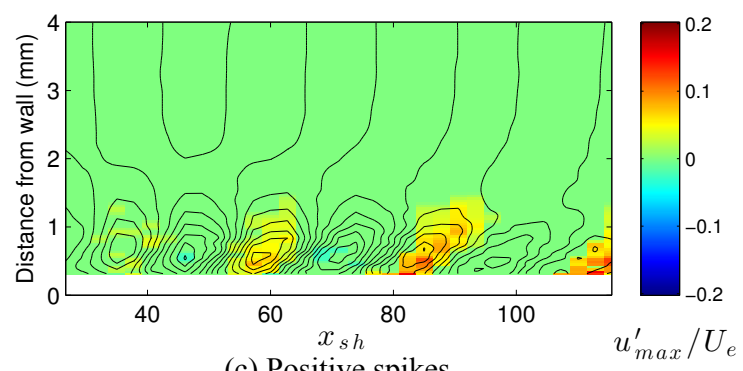

(c) Positive spikes

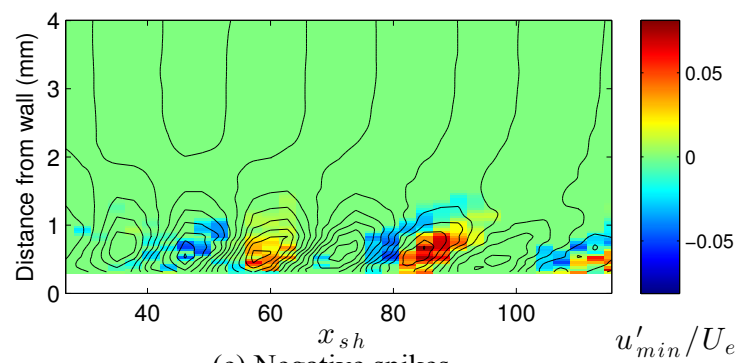

(e) Negative spikes

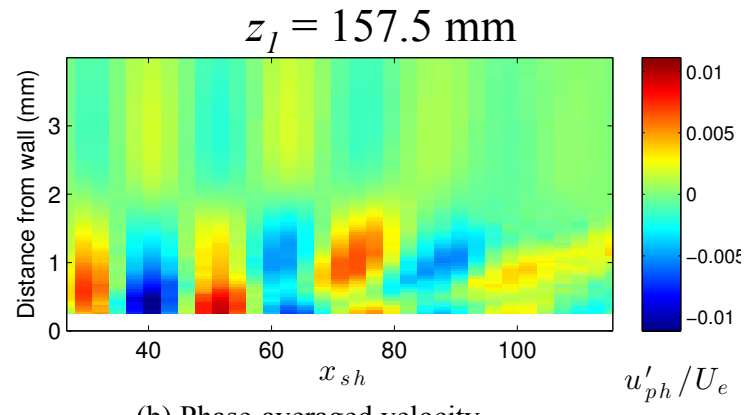

(b) Phase-averaged velocity

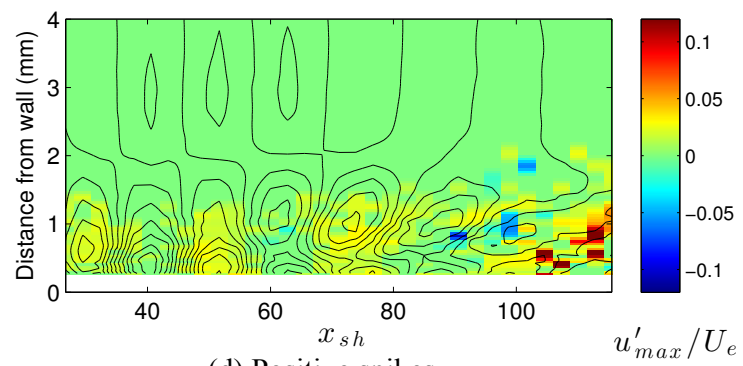

(d) Positive spikes

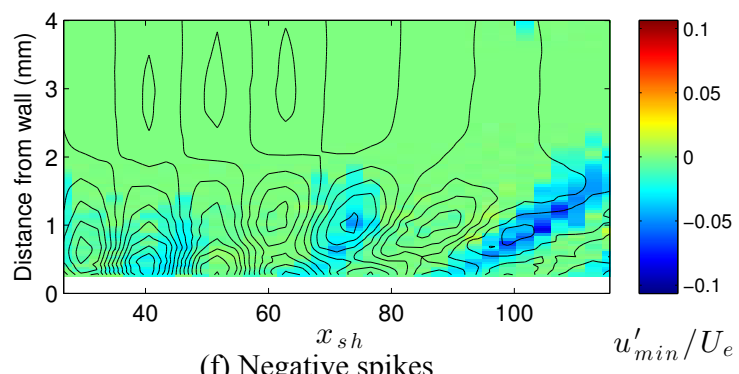

(f) Negative spikes

Figure 18: Phase-averaged xy-scan results with $300 \mathrm{~Hz}$ forcing, $t=0 \mathrm{~s}$. Contour lines of the phase-averaged velocity at the same location are overlaid on Figs. c-f 


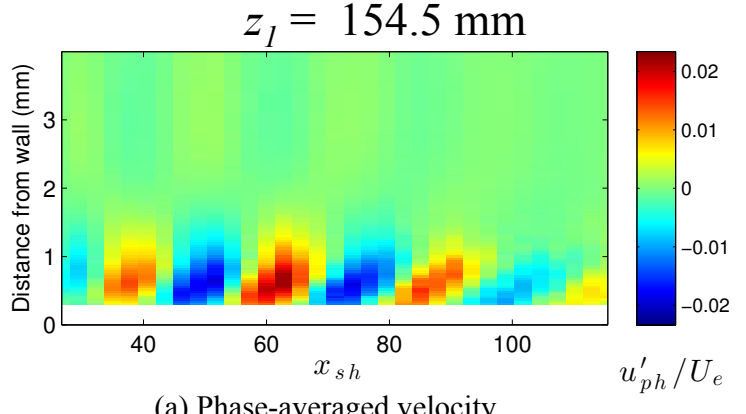

(a) Phase-averaged velocity

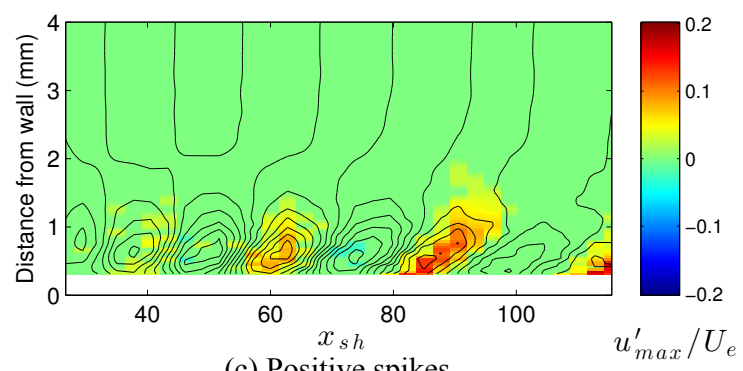

(c) Positive spikes

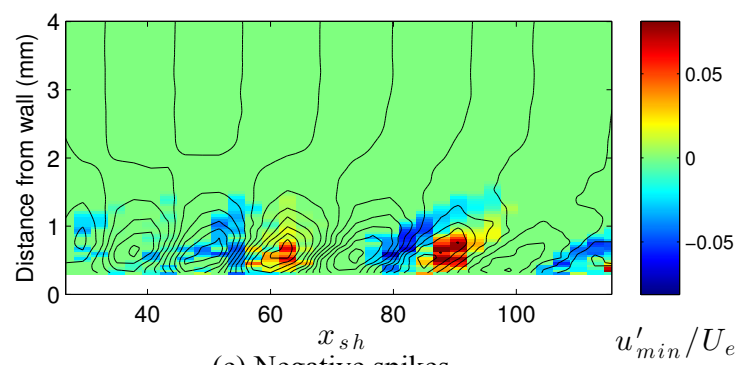

(e) Negative spikes

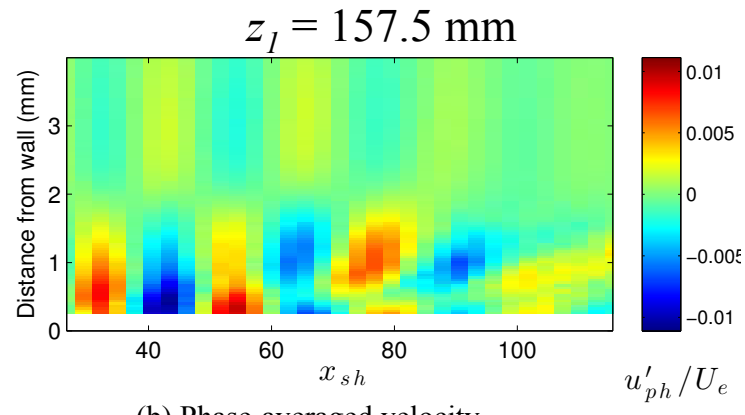

(b) Phase-averaged velocity

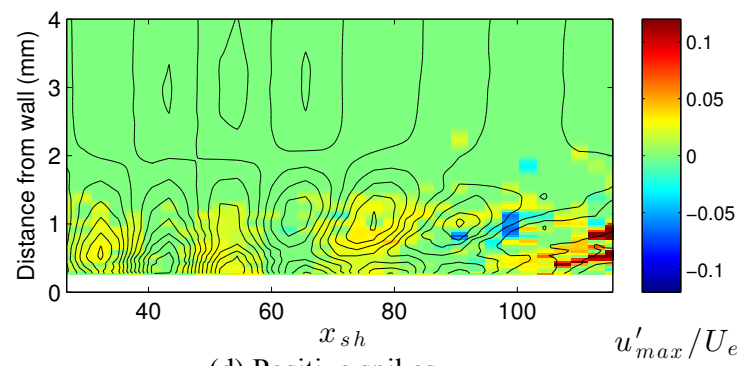

(d) Positive spikes

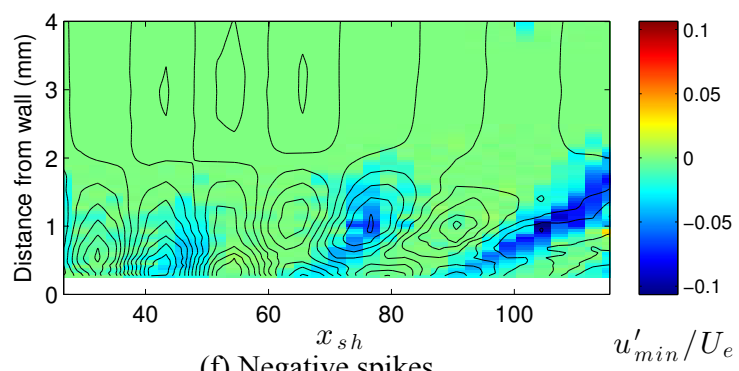

(f) Negative spikes

Figure 19: Phase-averaged xy-scan results with $300 \mathrm{~Hz}$ forcing, $t=3.67 \mathrm{e}-4 \mathrm{~s}$. Contour lines of the phaseaveraged velocity at the same location are overlaid on Figs. c-f 


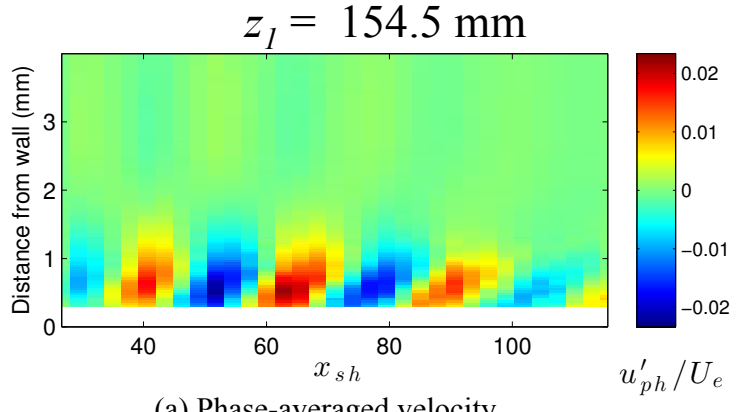

(a) Phase-averaged velocity

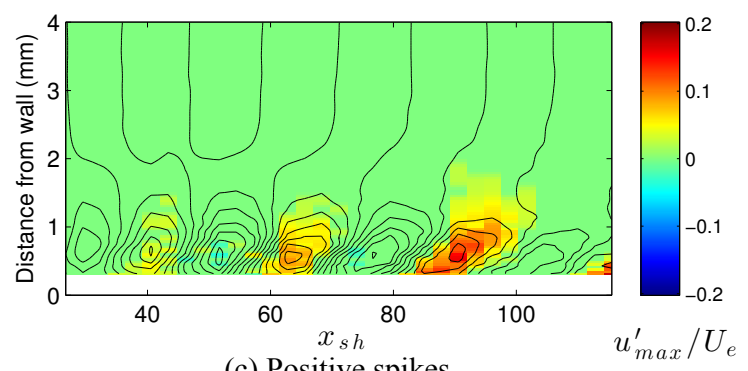

(c) Positive spikes

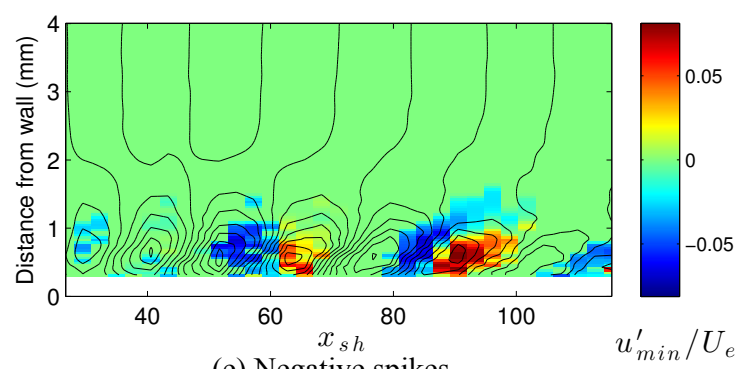

(e) Negative spikes

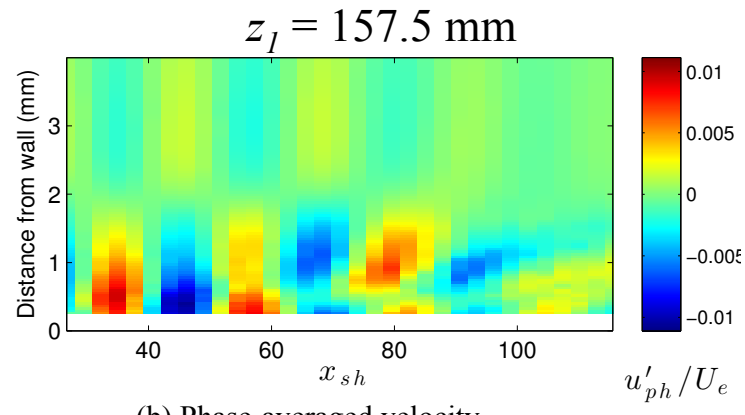

(b) Phase-averaged velocity

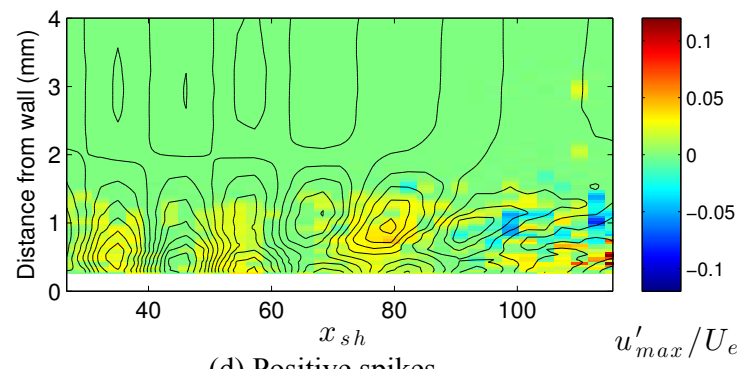

(d) Positive spikes

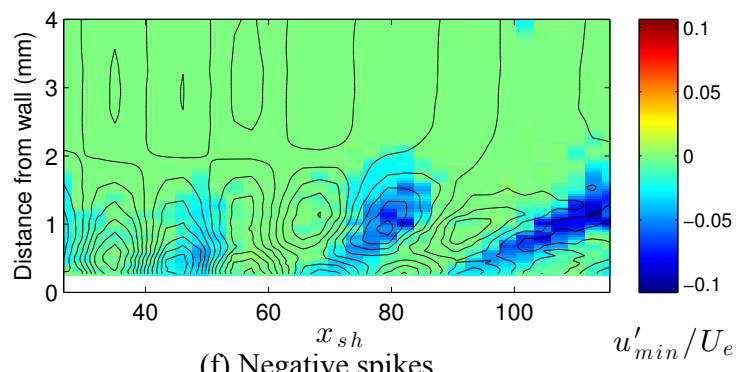

(f) Negative spikes

Figure 20: Phase-averaged xy-scan results with $300 \mathrm{~Hz}$ forcing, $t=7.33 \mathrm{e}-4 \mathrm{~s}$. Contour lines of the phaseaveraged velocity at the same location are overlaid on Figs. c-f 


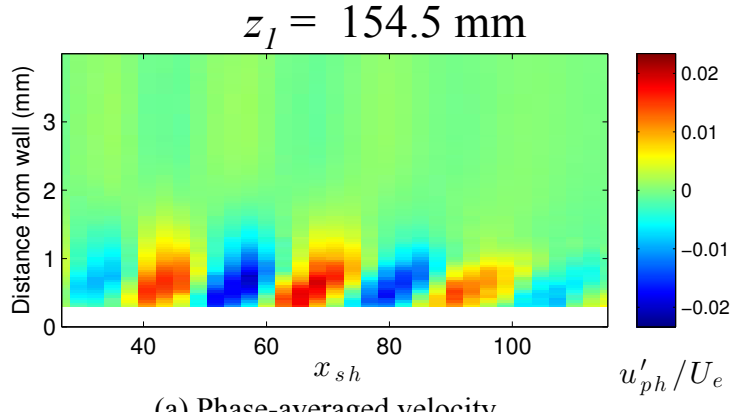

(a) Phase-averaged velocity

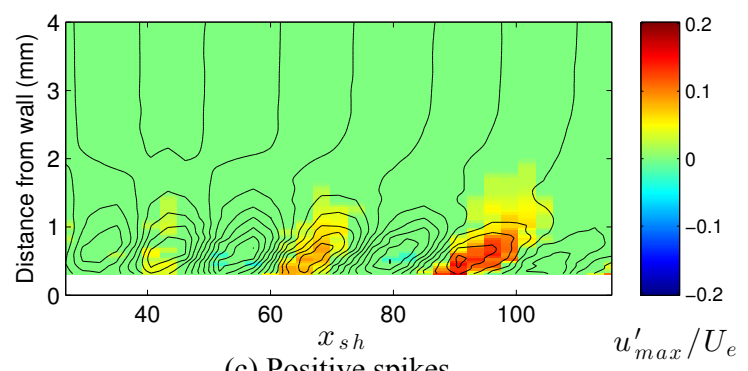

(c) Positive spikes

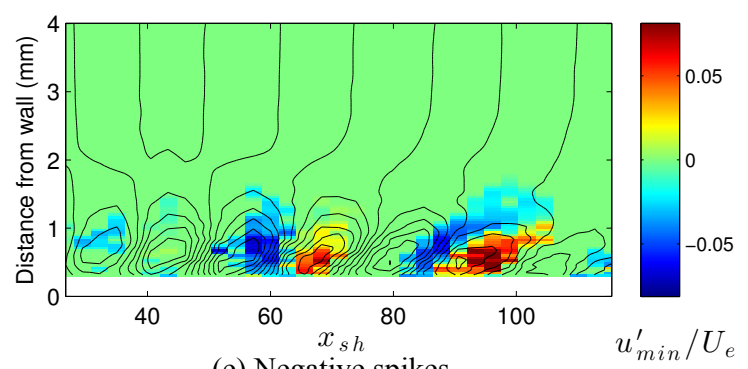

(e) Negative spikes

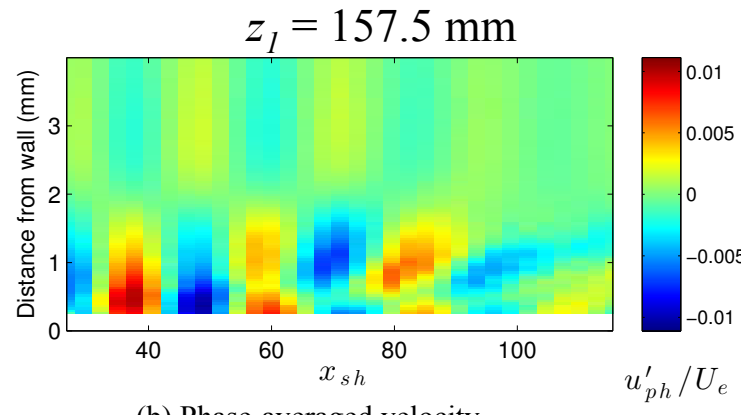

(b) Phase-averaged velocity

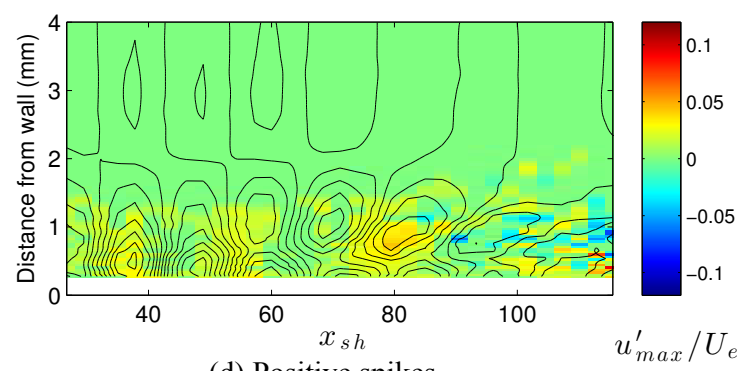

(d) Positive spikes

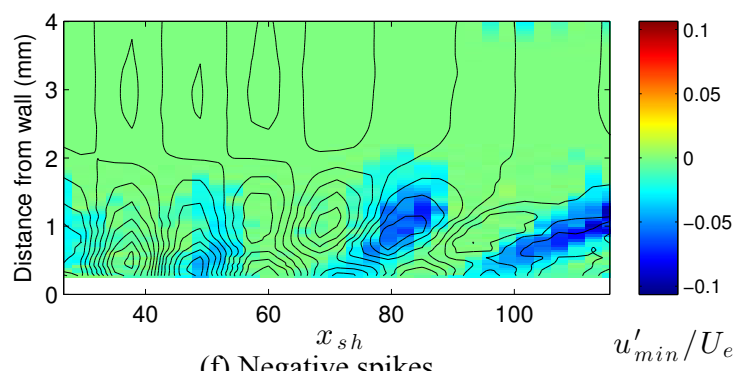

(f) Negative spikes

Figure 21: Phase-averaged xy-scan results with $300 \mathrm{~Hz}$ forcing, $t=1.1 \mathrm{e}-3 \mathrm{~s}$. Contour lines of the phaseaveraged velocity at the same location are overlaid on Figs. c-f 


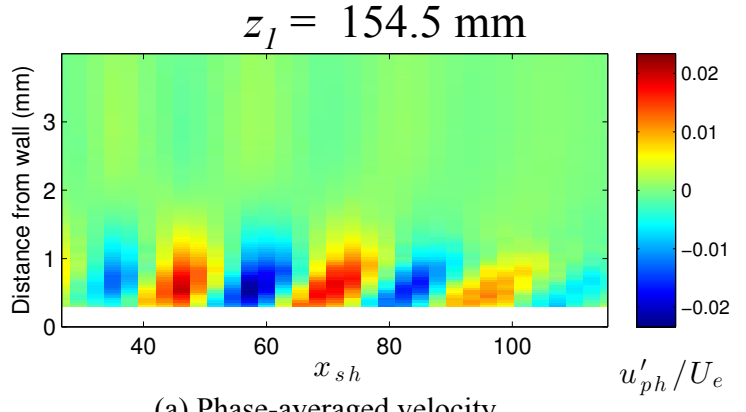

(a) Phase-averaged velocity

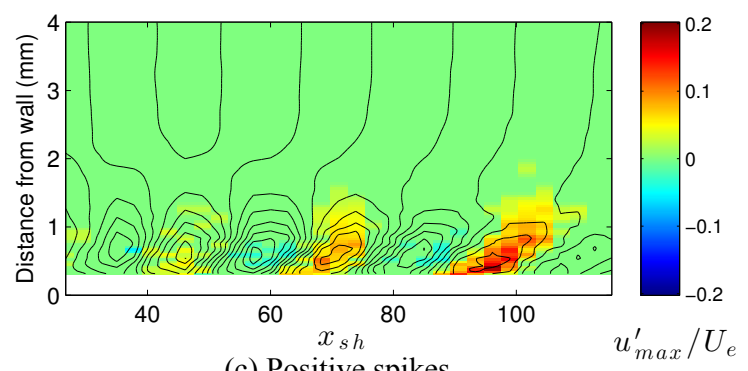

(c) Positive spikes

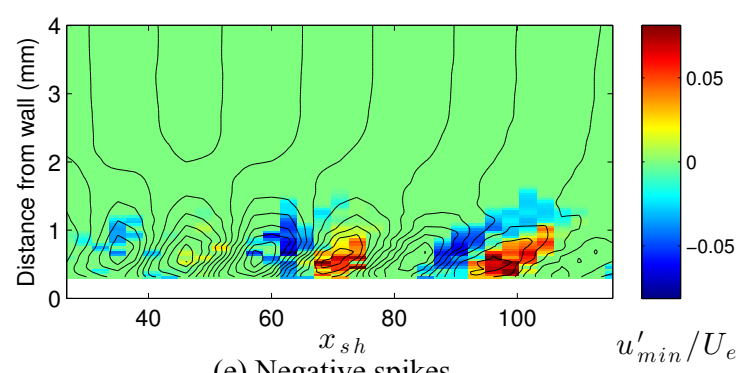

(e) Negative spikes

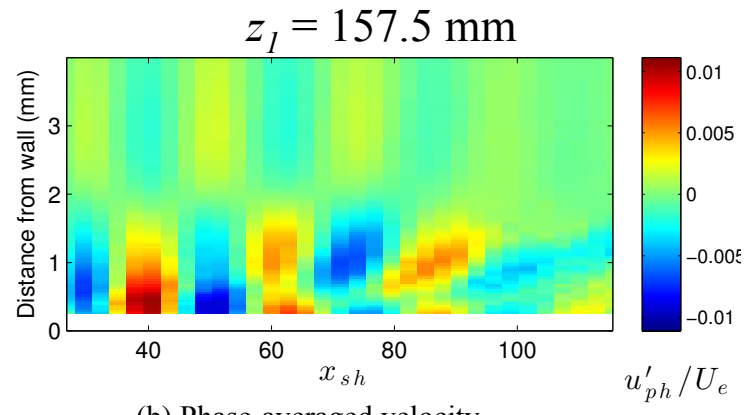

(b) Phase-averaged velocity

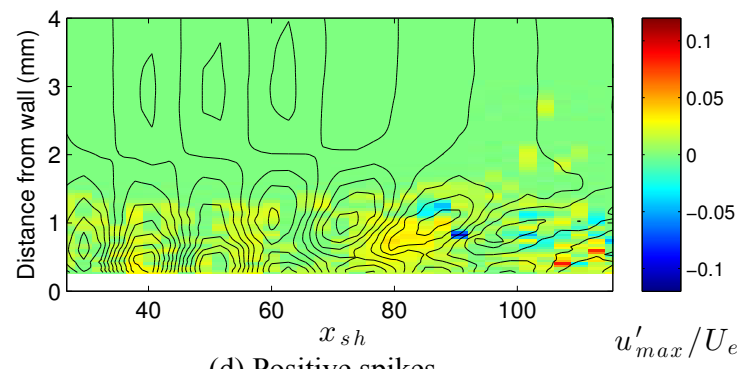

(d) Positive spikes

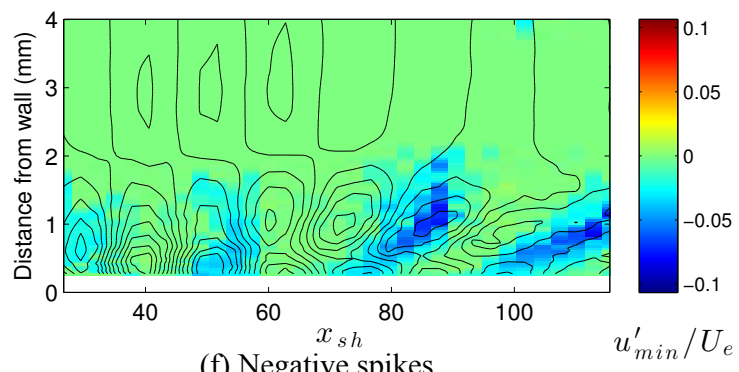

(f) Negative spikes

Figure 22: Phase-averaged xy-scan results with $300 \mathrm{~Hz}$ forcing, $t=1.5 \mathrm{e}-3 \mathrm{~s}$. Contour lines of the phaseaveraged velocity at the same location are overlaid on Figs. c-f 


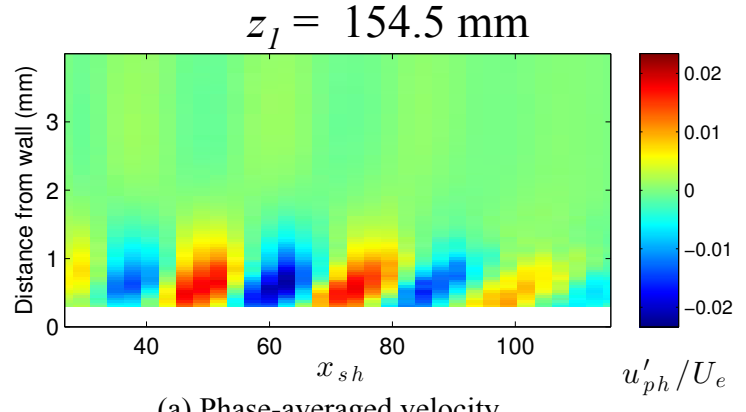

(a) Phase-averaged velocity

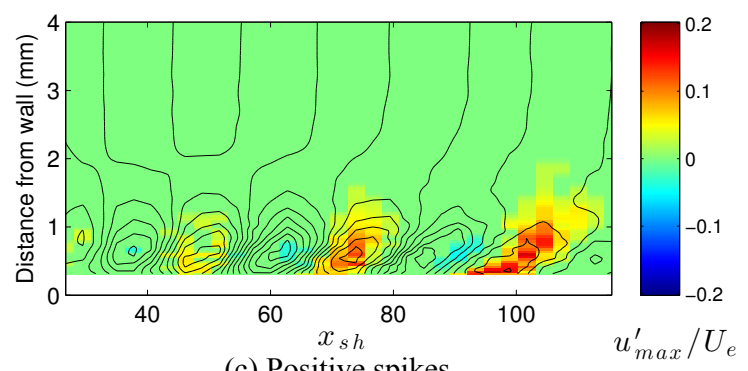

(c) Positive spikes

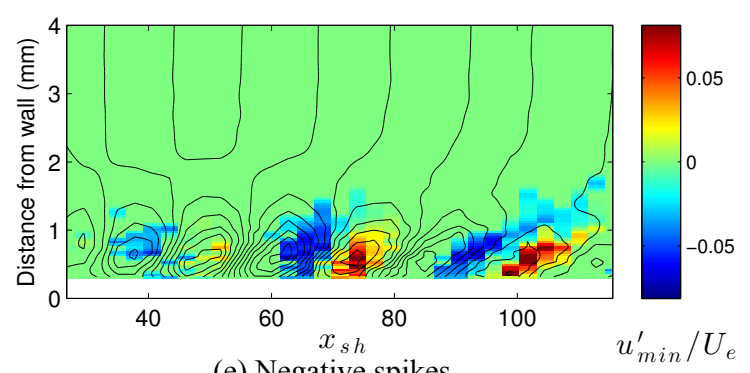

(e) Negative spikes

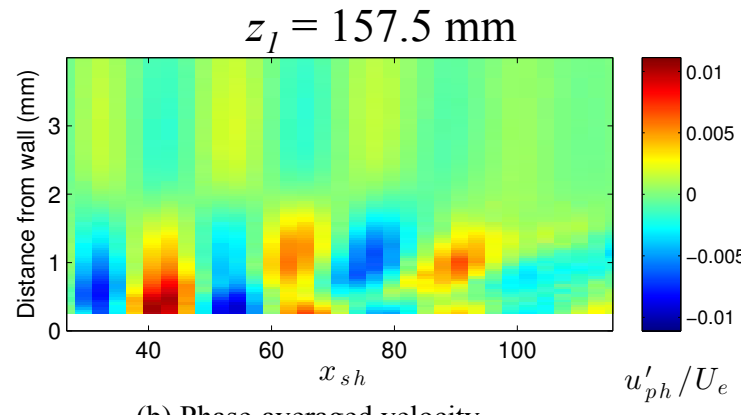

(b) Phase-averaged velocity

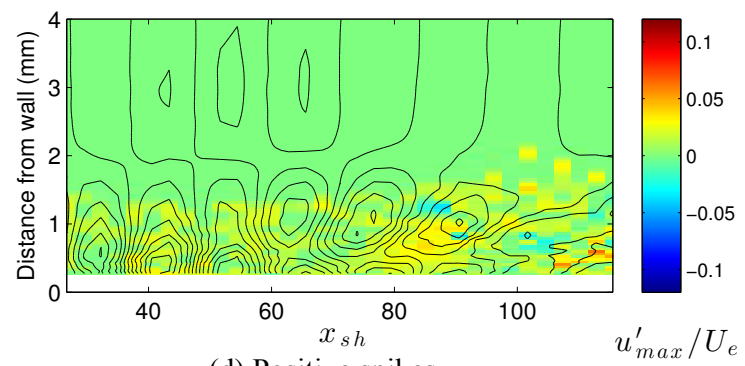

(d) Positive spikes

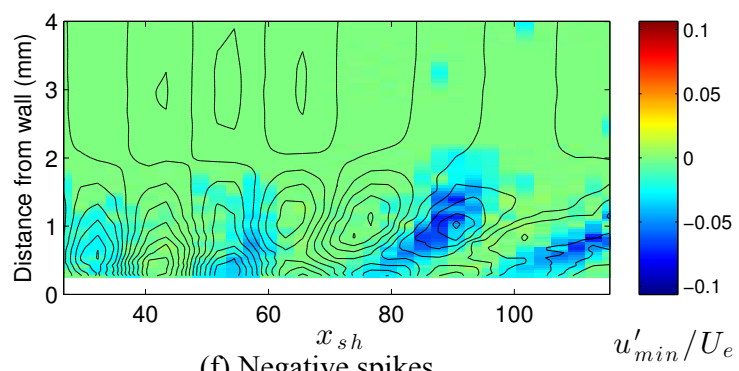

(f) Negative spikes

Figure 23: Phase-averaged xy-scan results with $300 \mathrm{~Hz}$ forcing, $t=1.8 \mathrm{e}-3 \mathrm{~s}$. Contour lines of the phaseaveraged velocity at the same location are overlaid on Figs. c-f 


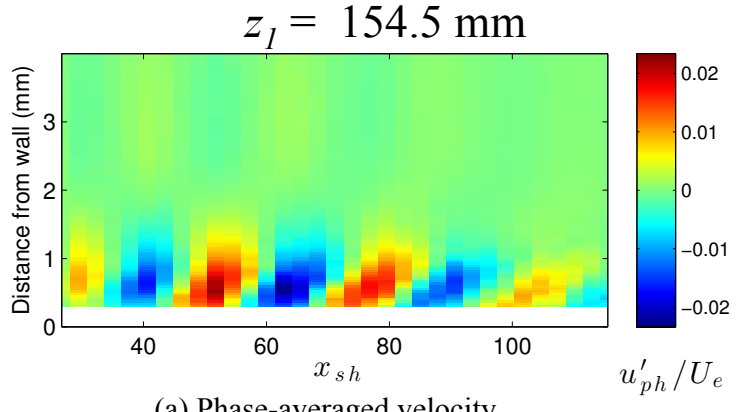

(a) Phase-averaged velocity

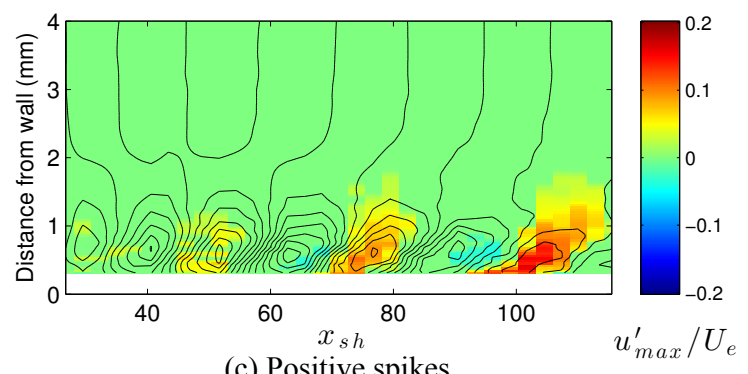

(c) Positive spikes

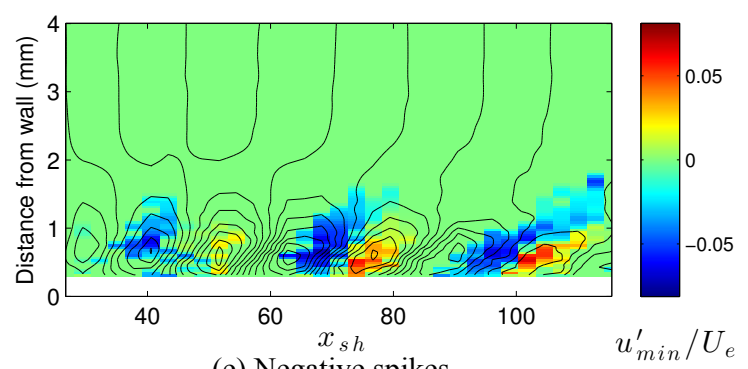

(e) Negative spikes

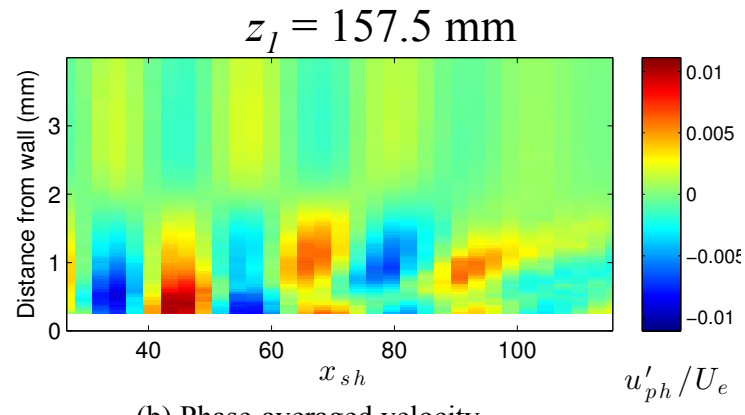

(b) Phase-averaged velocity

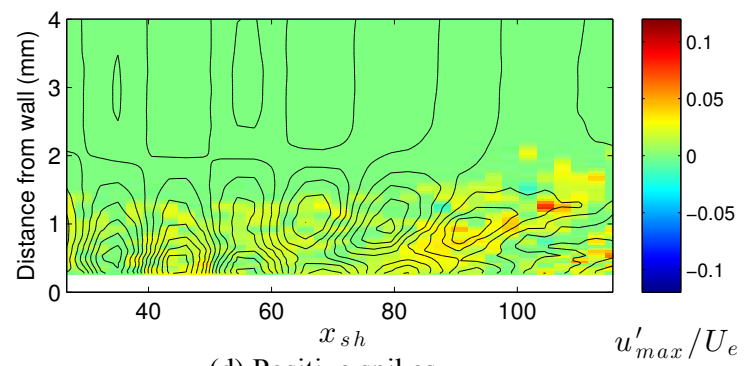

(d) Positive spikes

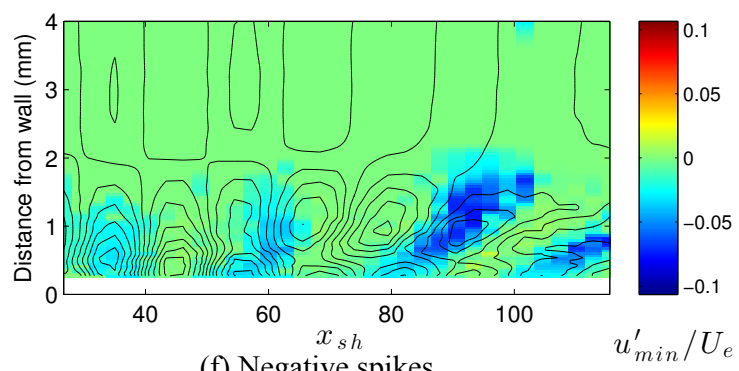

(f) Negative spikes

Figure 24: Phase-averaged xy-scan results with $300 \mathrm{~Hz}$ forcing, $t=2.2 \mathrm{e}-3 \mathrm{~s}$. Contour lines of the phaseaveraged velocity at the same location are overlaid on Figs. c-f 


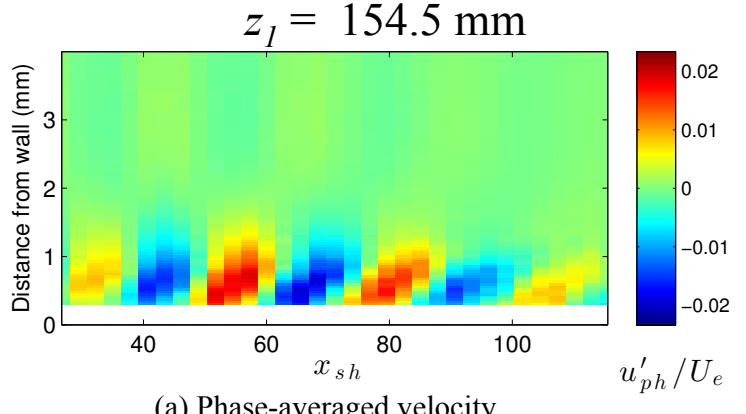

(a) Phase-averaged velocity

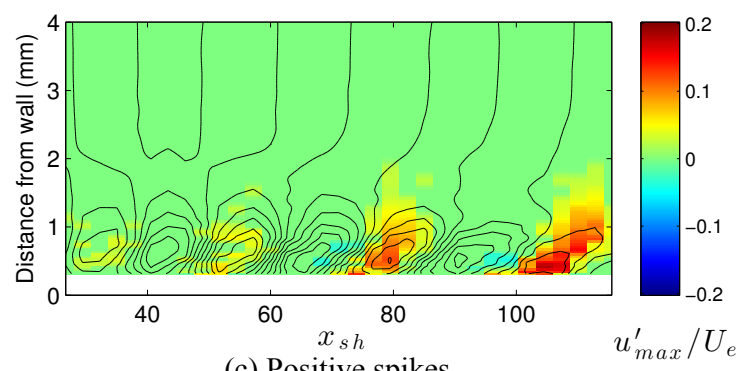

(c) Positive spikes

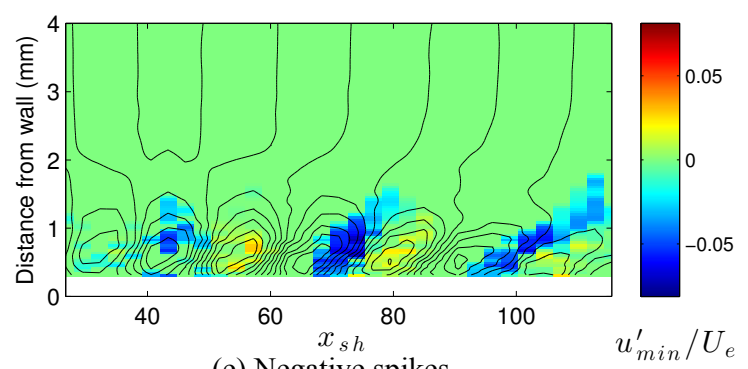

(e) Negative spikes

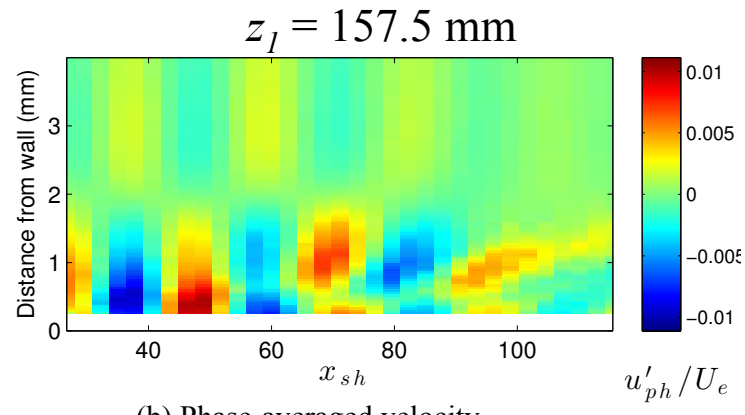

(b) Phase-averaged velocity

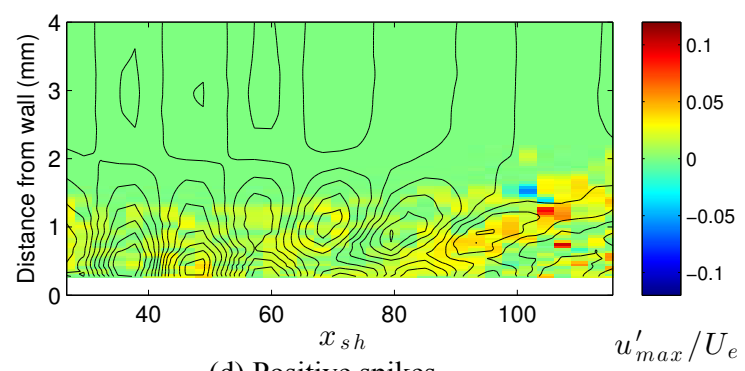

(d) Positive spikes

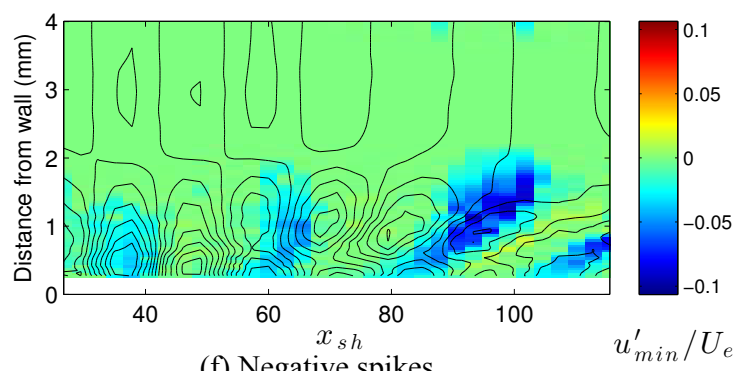

(f) Negative spikes

Figure 25: Phase-averaged xy-scan results with $300 \mathrm{~Hz}$ forcing, $t=2.6 \mathrm{e}-3 \mathrm{~s}$. Contour lines of the phaseaveraged velocity at the same location are overlaid on Figs. c-f 


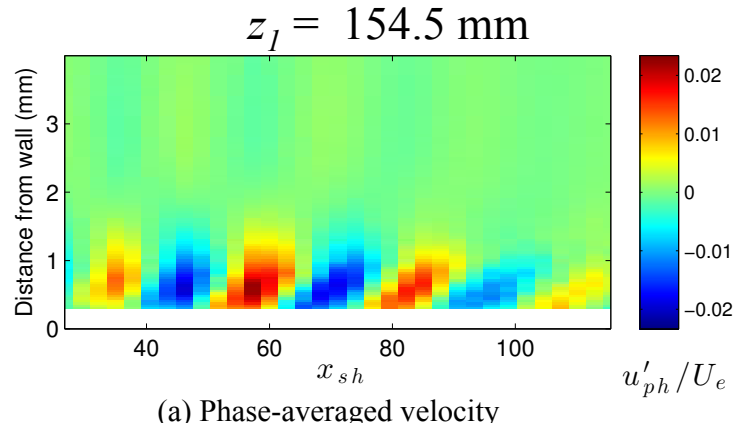

(a) Phase-averaged velocity

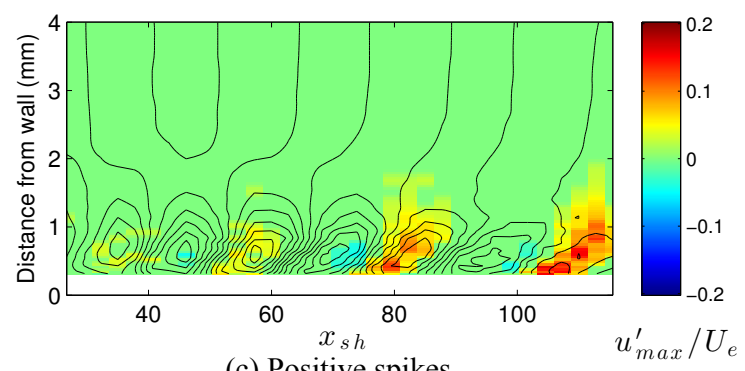

(c) Positive spikes

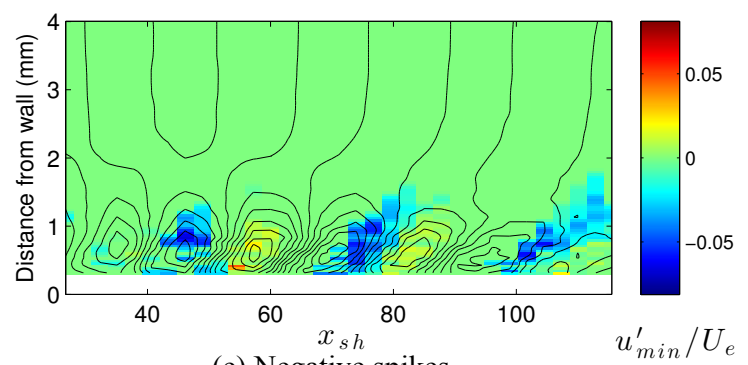

(e) Negative spikes

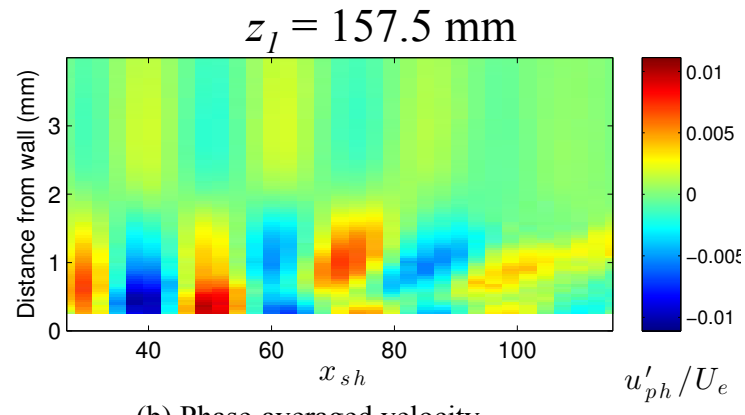

(b) Phase-averaged velocity

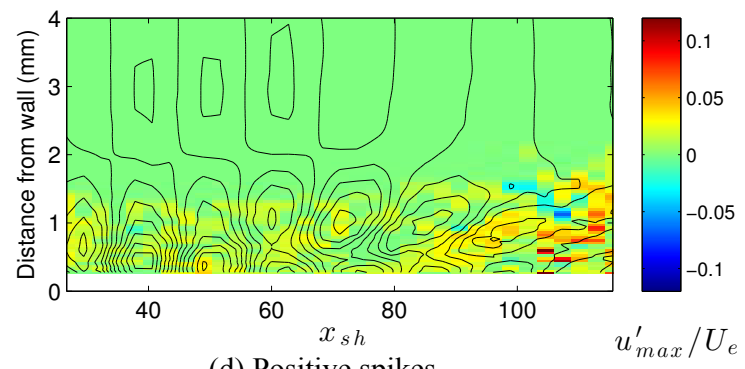

(d) Positive spikes

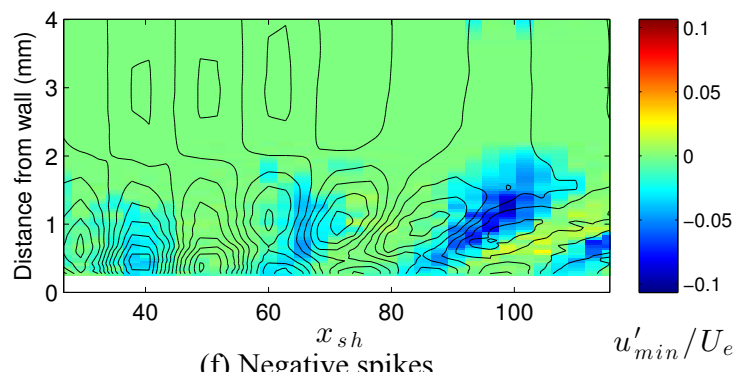

(f) Negative spikes

Figure 26: Phase-averaged xy-scan results with $300 \mathrm{~Hz}$ forcing, $t=2.9 \mathrm{e}-3 \mathrm{~s}$. Contour lines of the phaseaveraged velocity at the same location are overlaid on Figs. c-f 


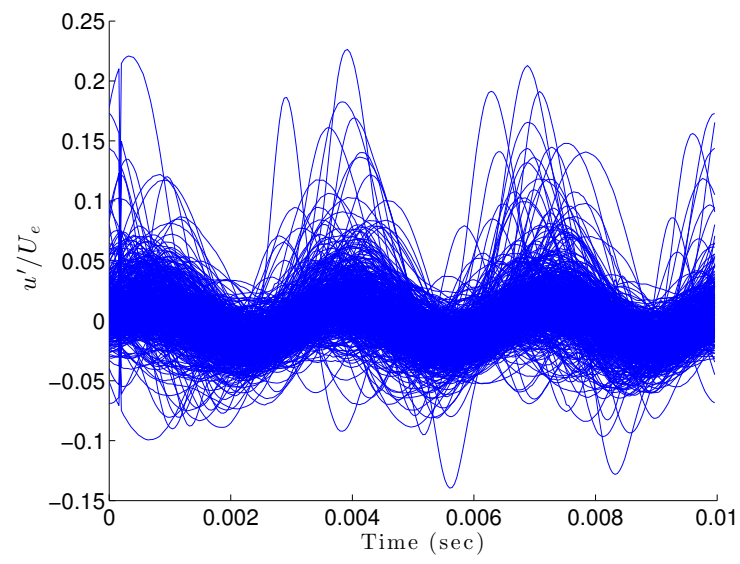

(a) Example of a long time segment showing the relationship of the positive spikes to the primary mode

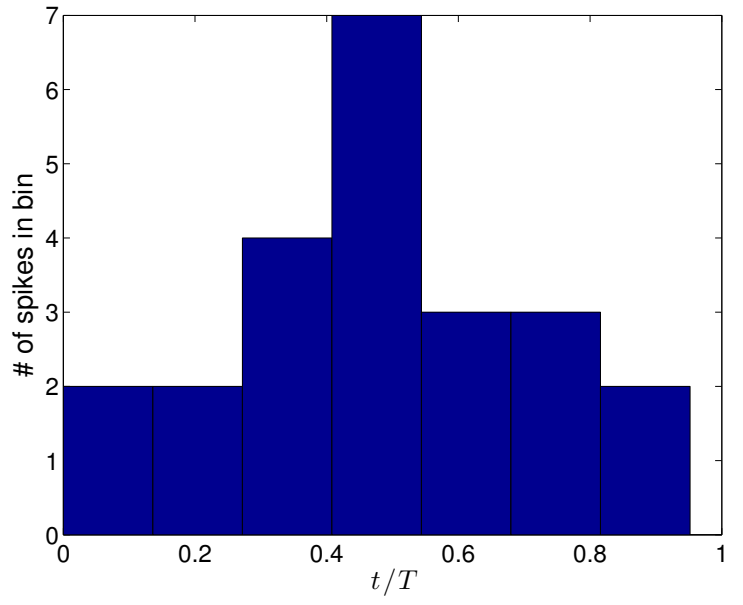

(b) Example of spike distribution vs. time

Figure 27: Examples of spike properties

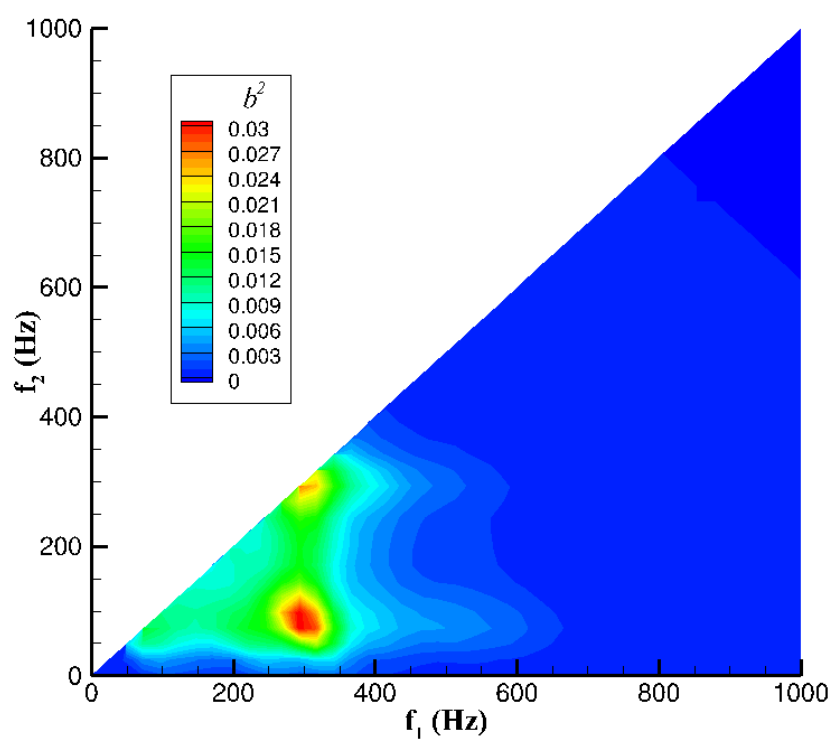

Figure 28: Auto-bicoherence at $x_{s h}=82, z=157 \mathrm{~mm}, y=0.45 \mathrm{~mm}$ 


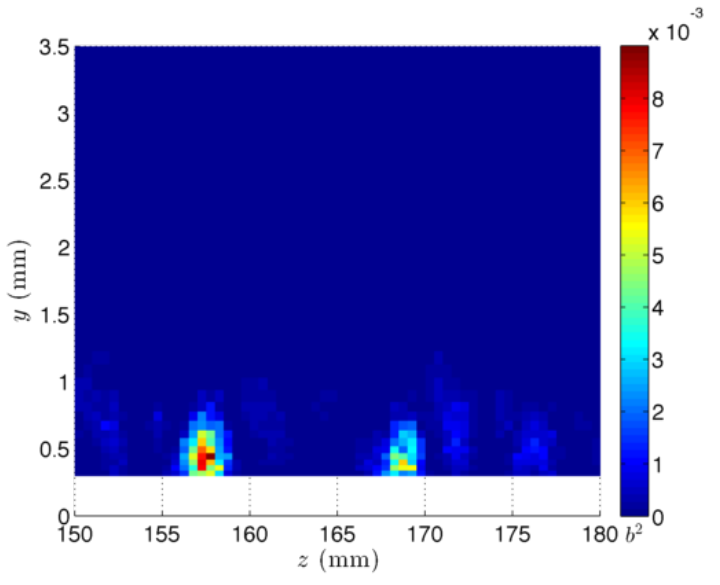

(a) $x_{s h}=60$

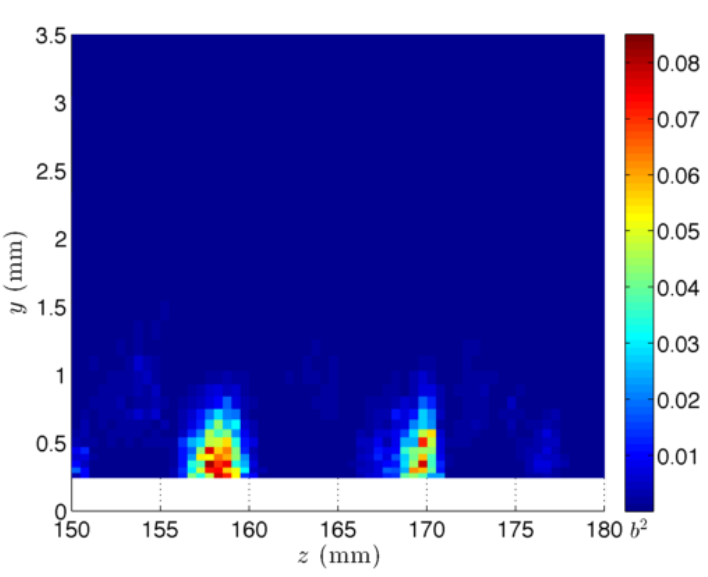

(b) $x_{s h}=82$

Figure 29: Auto-bicoherence of $100 \mathrm{~Hz}$ and $300 \mathrm{~Hz}$

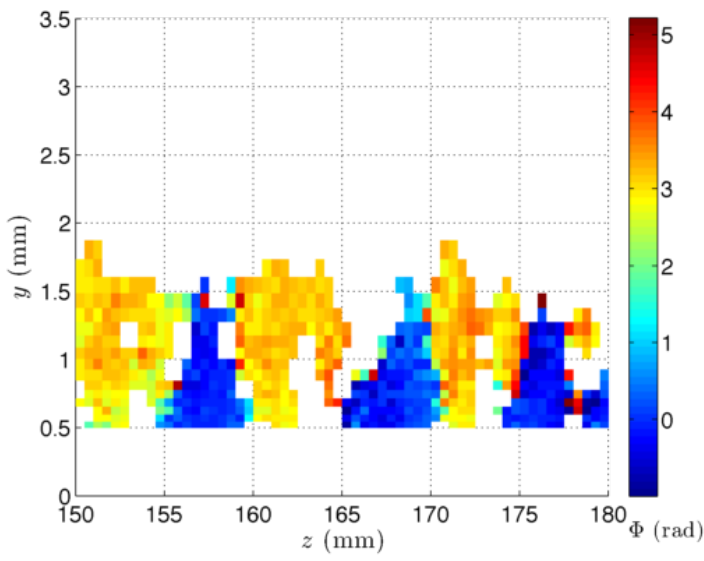

(a) $x_{s h}=60$

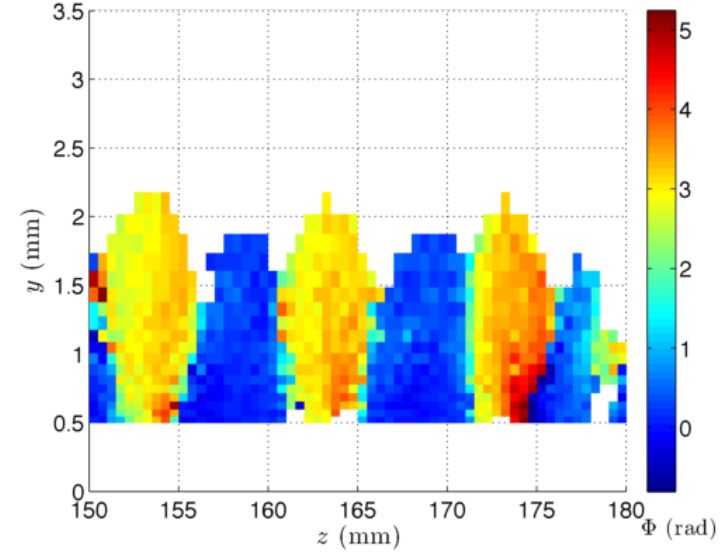

(b) $x_{s h}=82$

Figure 30: Biphase of $100 \mathrm{~Hz}$ and $300 \mathrm{~Hz}$

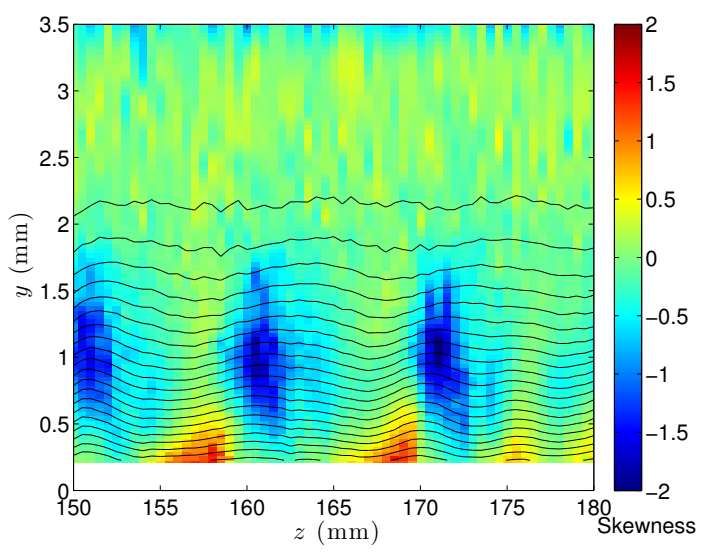

(a) $x_{s h}=60$

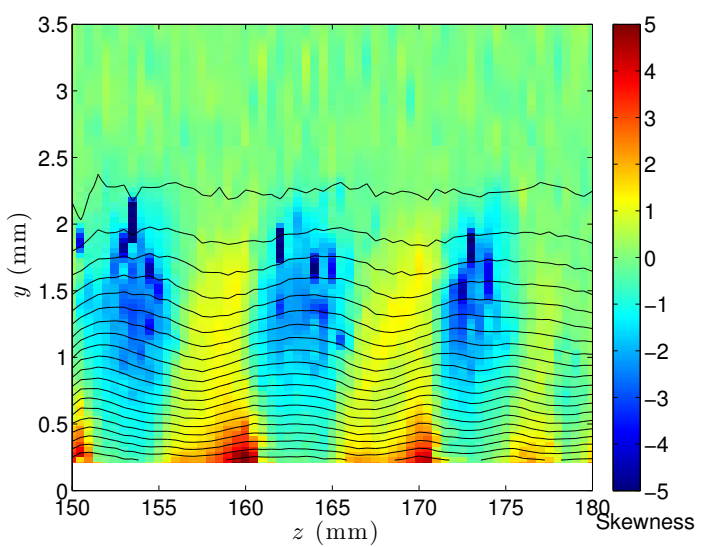

(b) $x_{s h}=82$

Figure 31: Skewness contours (colors) overlaid with mean flow contours (lines), showing locations of positive and negative spikes 


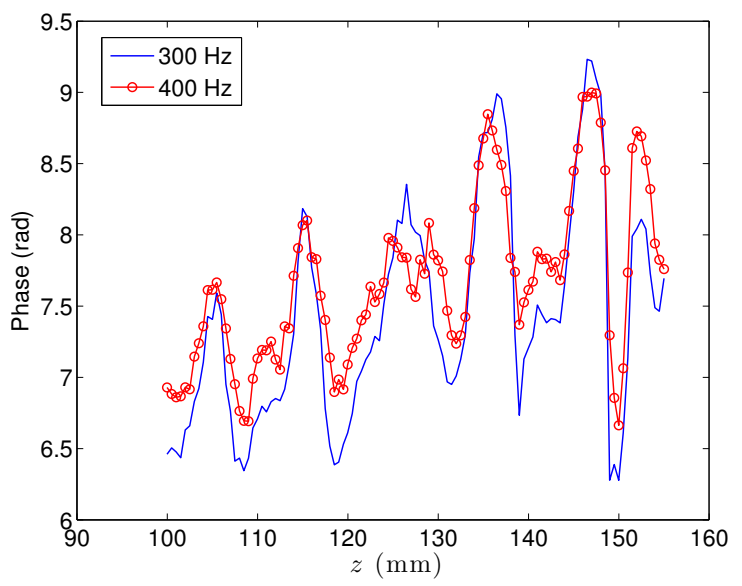

(a) $z$-scan

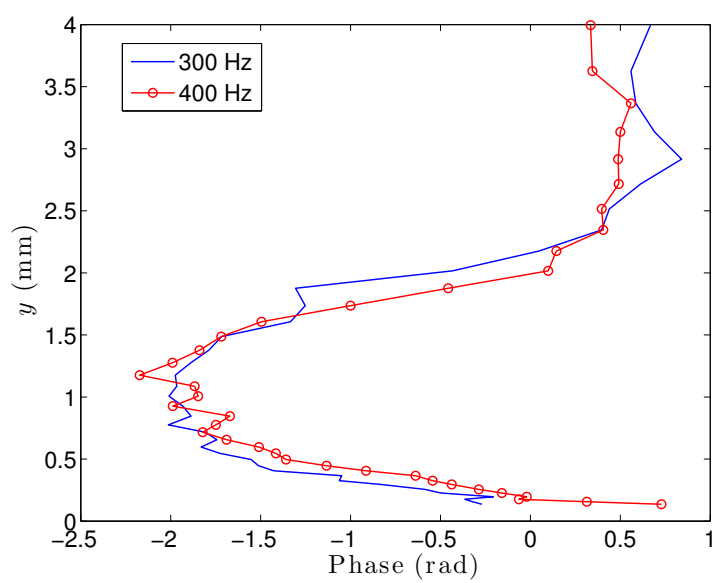

(b) Single profile

Figure 32: Phase plots comparing 300 and $400 \mathrm{~Hz}$ at $x_{s h} \approx 60$

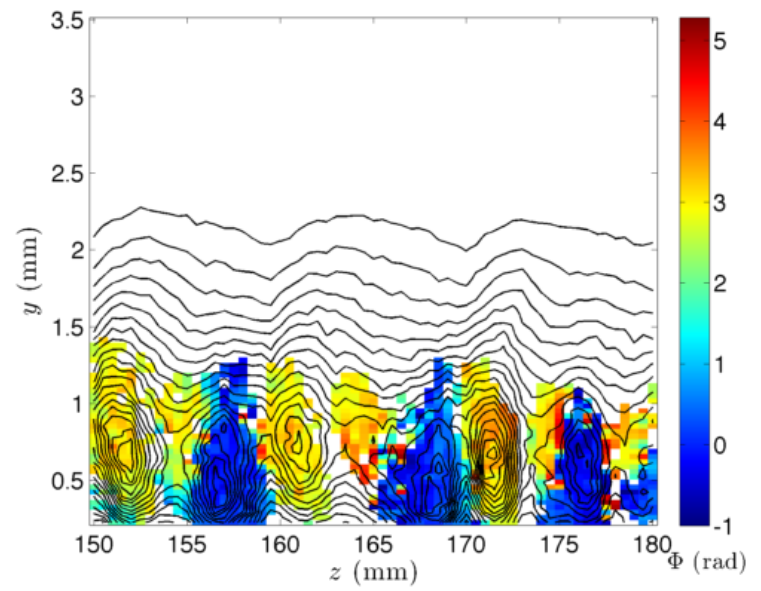

(a) $x_{s h}=60$

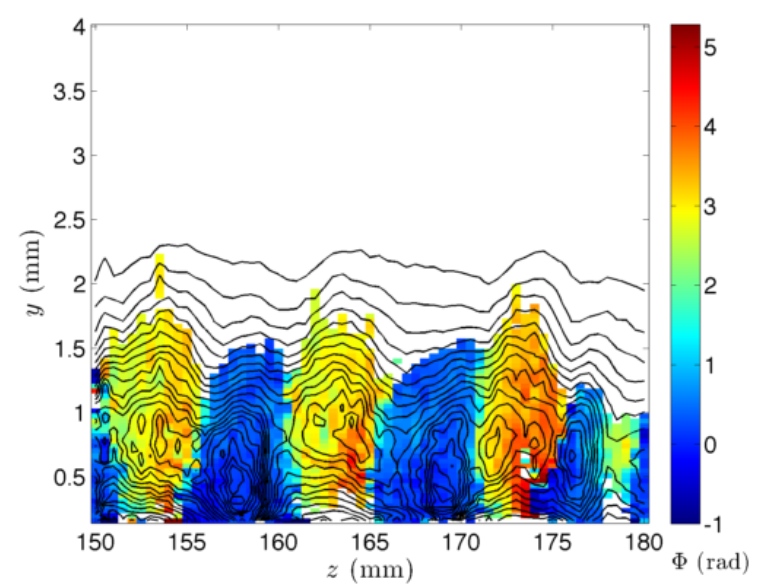

(b) $x_{s h}=82$

Figure 33: Biphase of 100 and $300 \mathrm{~Hz}$ (colors) overlaid with contours of $u_{r m s}^{\prime}$ for $f=80$ to $200 \mathrm{~Hz}$ (traveling crossflow frequency band) 


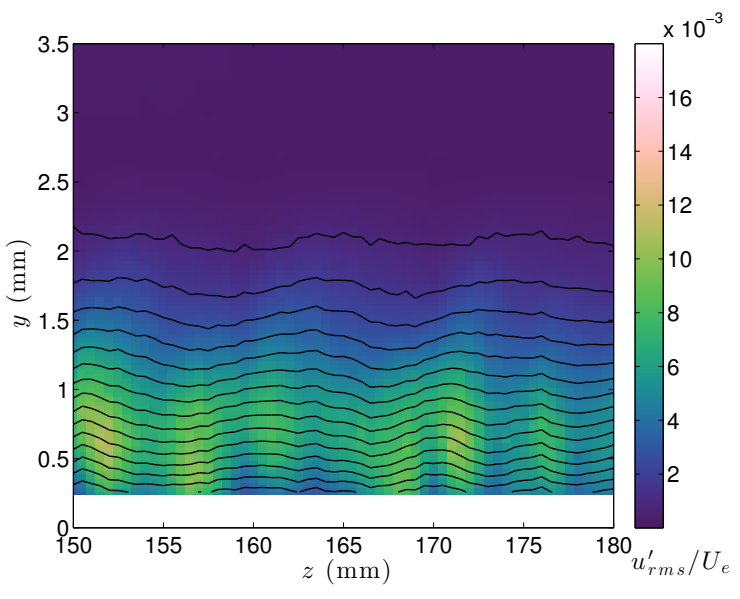

(a) Traveling crossflow instability, $x_{s h}=60$

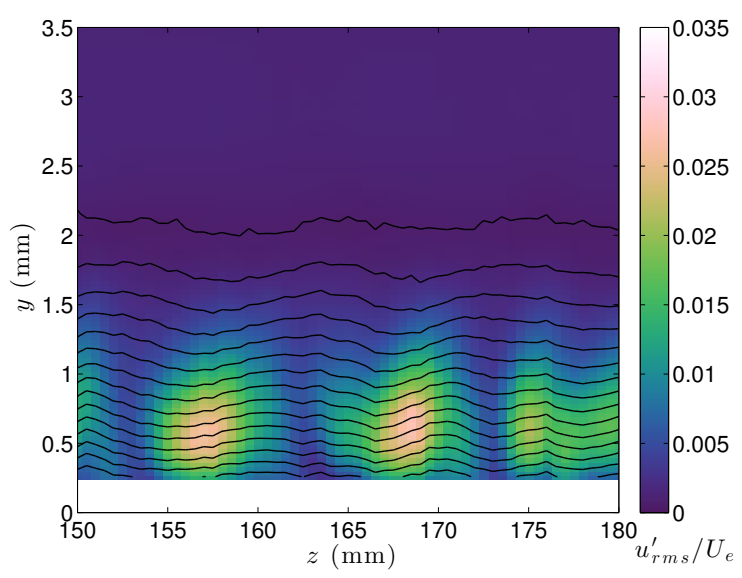

(c) TS instability, $x_{s h}=60$

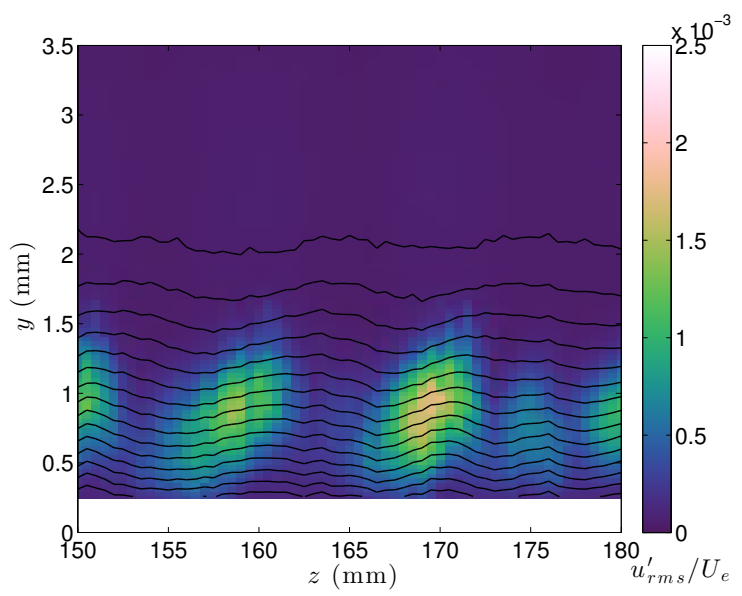

(e) Shear-layer instability, $x_{s h}=60$

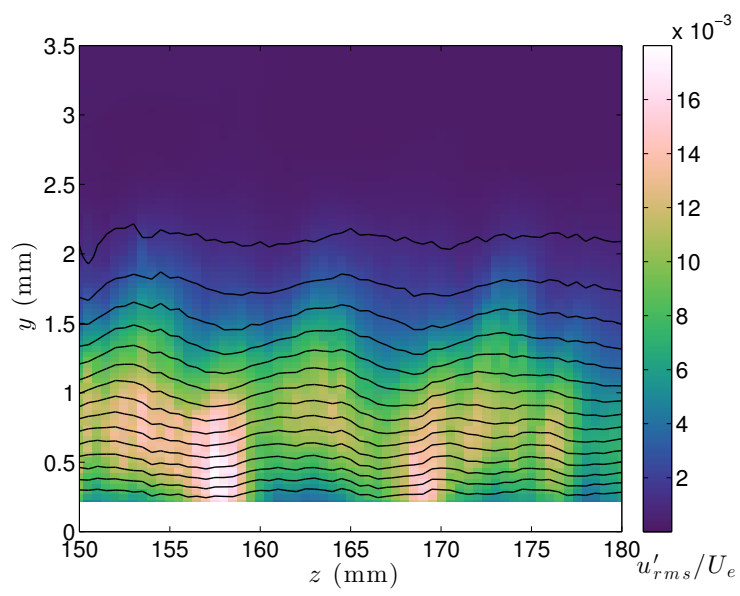

(b) Traveling crossflow instability, $x_{s h}=82$

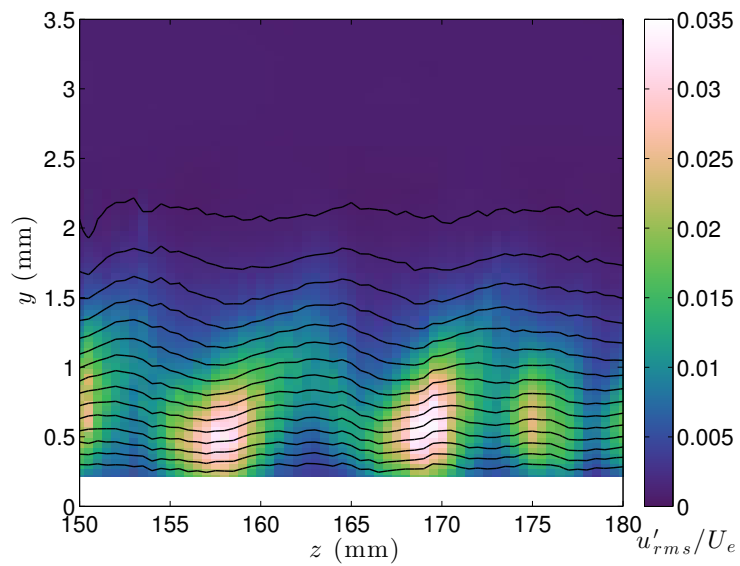

(d) TS instability, $x_{s h}=82$

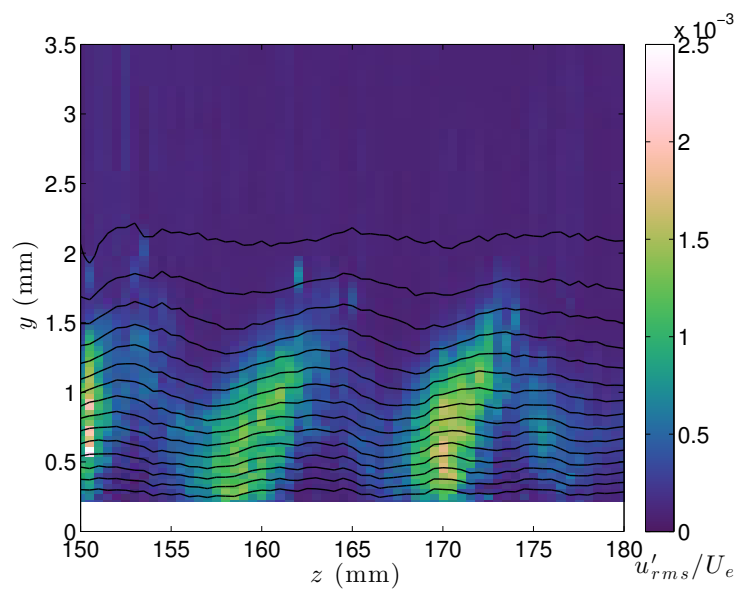

(f) Shear-layer instability, $x_{s h}=82$

Figure 34: Comparison of all disturbance bands $u_{r m s}^{\prime}$ (colors) with forcing at $f=300 \mathrm{~Hz}$, overlaid with mean-flow contours (lines) 